\title{
The distribution of nearby stars in phase space mapped by Hipparcos ${ }^{\star}$
}

\section{Clustering and streaming among A-F type stars}

\author{
E. Chereul ${ }^{1}$, M. Crézé $e^{1,2}$, and O. Bienaymé ${ }^{1}$ \\ 1 Centre de Données de Strasbourg, Observatoire Astronomique de Strasbourg, 11 rue de l'Université, \\ F-67000 Strasbourg, France \\ 2 I.U.P de Vannes, Tohannic, rue Yves Mainguy, F-56000 Vannes, France
}

Received April 21; accepted September 21, 1998

\begin{abstract}
This paper presents the detailed results obtained in the search of density-velocity inhomogeneities in a volume limited and absolute magnitude limited sample of A-F type dwarfs within 125 parsecs of the Sun. A 3 -D wavelet analysis is used to extract inhomogeneities, both in the density and velocity distributions. Thus, a real picture of the phase space is produced. Not only are some clusters and streams detected, but the fraction of clumped stars can be measured. By estimating individual stellar ages one can relate the streams and clusters to the state of the interstellar medium (ISM) at star formation time and provide a quantitative view of cluster evaporation and stream mixing.

Having established this picture without assumption we come back to previously known observational facts regarding clusters and associations, moving groups or so-called superclusters.
\end{abstract}

In the $3-\mathrm{D}$ position space, well known open clusters (Hyades, Coma Berenices and Ursa Major), associations (parts of the Scorpio-Centaurus association) as well as the Hyades evaporation track are retrieved. Three new probably loose clusters are identified (Bootes, Pegasus 1 and 2). The sample is relatively well mixed in the position space since less than 7 per cent of the stars belong to structures with coherent kinematics, most likely gravitationally bound.

The wavelet analysis exhibits strong velocity structuring at typical scales of velocity dispersion $\bar{\sigma}_{\text {stream }} \sim 6.3$, 3.8 and $2.4 \mathrm{~km} \mathrm{~s}^{-1}$. The majority of large scale velocity structures $\left(\bar{\sigma}_{\text {stream }} \sim 6.3 \mathrm{~km} \mathrm{~s}^{-1}\right)$ are Eggen's superclusters (Pleiades SCl, Hyades SCl and Sirius SCl) with the whole Centaurus association. A new supercluster-like structure is found with a mean velocity between the Sun

Send offprint requests to: chereul@cdsxb6.u-strasbg.fr

* Based on data from the Hipparcos astrometry satellite. and Sirius SCl velocities. These structures are all characterized by a large age range which reflects the overall sample age distribution. Moreover, a few old streams of $\sim 2$ Gyr are also extracted at this scale with high $U$ components. We show that all these large velocity dispersion structures represent $63 \%$ of the sample. This percentage drops to $46 \%$ if we remove the velocity background created by a smooth velocity ellipsoid in each structure. Smaller scales $\left(\bar{\sigma}_{\text {stream }} \sim 3.8\right.$ and $\left.2.4 \mathrm{~km} \mathrm{~s}^{-1}\right)$ reveal that superclusters are always substructured by 2 or more streams which generally exhibit a coherent age distribution. At these scales, background stars are negligible and percentages of stars in streams are $38 \%$ and $18 \%$ respectively.

Key words: methods: data analysis, Galaxy: solar neighbourhood - galaxies open clusters and associations - galaxies: kinematics and dynamics

\section{Introduction}

The context of this study as well as implications of the results for the solar circle kinematics understanding are in Chereul et al. (1998) (hereafter Paper II). This third paper aims at providing to the reader of Paper II the details of the phase space inhomogeneities brought to the fore by means of wavelet analysis and technical details on several features of the analysis.

Wavelet analysis is extensively used to extract deviations from smooth homogeneity, both in density (clustering) and velocity distribution (streaming). A 3-D wavelet analysis tool is first developed and calibrated to recognize physical inhomogeneities among random fluctuations. It is applied separately in the position space (Sect. 4) and in the velocity space (Sect. 5). Once significant features are 
identified (whether in density or in velocity), feature members can be identified and their behaviour can be traced in the complementary space (velocity or density). Thus, a real picture of the phase space is produced. Not only are some clusters and streams detected, but the fraction of stars involved in clumpiness can be measured. Then, estimated stellar ages help connecting streams and clusters to the state of the ISM at star formation time and providing a quantitative view of cluster melting and stream mixing at work.

Only once this picture has been established on a priorless basis we come back to previously known observational facts such as clusters and associations, moving groups or so-called superclusters. A sine qua non condition for this analysis to make sense is the completeness of data within well defined limits. In so far as positions, proper motions, distances, magnitudes and colours are concerned, the Hipparcos mission did care. Things are unfortunately not so simple concerning radial velocities and ages. More than half radial velocities are missing and the situation regarding Strömgren photometry data for age estimation is not much better. So we developed palliative methods which are calibrated and tested on available true data to circumvent the completeness failure. The palliative for radial velocities is based on an original combination of the classical convergent point method (Sect. 5.1) with the wavelet analysis. The palliative for ages (Sect. 3) is an empirical relationship between age and an absolutemagnitude/colour index. It has only statistical significance in a very limited range of the HR diagram.

The sample was pre-selected inside the Hipparcos Input Catalogue (ESA, 1992) among the "Survey stars". The limiting magnitude is $m_{v} \leq 7.9+1.1 \cdot \sin |b|$ for spectral types earlier than G5 (Turon et al. 1992). Spectral types from A0 to G0 with luminosity classes V and VI were kept. Within this pre-selection the final choice was based on Hipparcos (ESA, 1997) magnitude $\left(m_{v} \leq 8.0\right)$, colours $(-0.1 \leq B-V \leq 0.6)$, and parallaxes $(\pi \geq 8$ mas $)$. The sample studied (see sample named h125 in Paper I, Crézé et al. 1998) is a slice in absolute magnitude of this selection containing 2977 A-F type dwarf stars with absolute magnitudes brighter than 2.5. It is complete within 125 pc from the Sun.

Details concerning the wavelet analysis implementation through the "à trou" algorithm and the thresholding procedure are given in Sect. 2. The palliative age determination method is fully described in Sect. 3. The results of the density analysis in position space (clustering) are given in Sect. 4. The convergent point method is explained in Sect. 5.1 and the procedures estimating spurious members and field stars among detected streams are given in Sects. 5.2.1 and 5.2.2 respectively. The results of the velocity space analysis (streaming) are in Sect. 5.3 including a detailed review of observational facts and a comparison with evidences collected by previous investigators. Conclusion in Sect. 6 summarizes the different results.
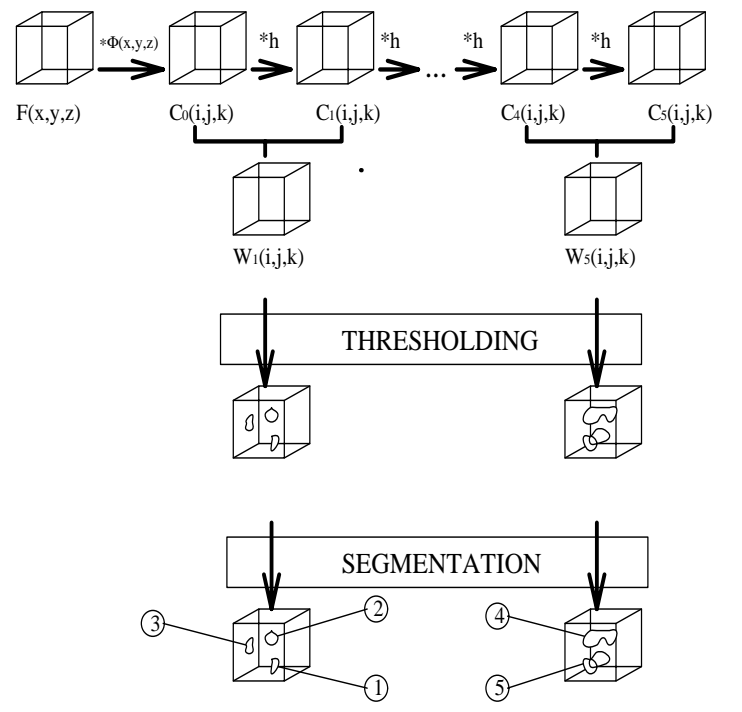

Fig. 1. Implementation of the wavelet analysis

\section{Implementation of the wavelet analysis}

Main reasons for the choice of this algorithm developed by Holschneider et al. (1989) as well as theoretical way to compute wavelet coefficients are provided in Paper II. Here we focus on the practical computation of the wavelet coefficients (Sect. 2.1) and the thresholding procedure adopted (Sects. 2.2 and 2.3).

\subsection{The "à trou" algorithm}

The adopted mother wavelet $\Psi(x)$ is defined as the difference at two different scales of a same scaling function $\Phi(x)$ (or smoothing function):

$\Psi(x)=\Phi(x)-\frac{1}{2} \Phi\left(\frac{x}{2}\right)$.

This particularity allows to use the "à trou" algorithm implementation of the wavelet transform. This algorithm avoids the direct computation of the scalar product between $\Psi(x)$ and the signal $F(x)$ to obtain wavelet coefficients. It uses an iterative schema based both on the relation existing between the mother wavelet and the scaling function (Eq. (1)) and the following relation

$\frac{1}{2^{s+1}} \Phi\left(\frac{x}{2^{s+1}}\right)=\sum_{l=-2}^{2} h(l) \Phi\left(\frac{x}{2^{s}}-l\right)$

where $h(l)=\left\{\frac{1}{16}, \frac{1}{4}, \frac{3}{8}, \frac{1}{4}, \frac{1}{16}\right\}$ is a one-dimensional discrete low-pass filter. $h(l)$ is applied in the algorithm to compute iteratively the different approximations (or smoothing) $C_{s}$ of the signal at each scale $s$. For a 3D signal like our density or velocity distributions, $h(l)$ is applied separately in each 
dimension to obtain the signal approximation at scale $s$ and pixel $(i, j, k)$ :

$$
\begin{array}{r}
C_{s}(i, j, k)=\sum_{l=-2}^{2} \sum_{m=-2}^{2} \sum_{n=-2}^{2} h(l) h(m) h(n) \\
\cdot C_{s-1}\left(i+2^{s-1} l, j+2^{s-1} m, k+2^{s-1} n\right) .
\end{array}
$$

The distance between two bins increases by a factor 2 from scale $s-1$ to scale $s$. The result at each scale $s$ $\left(C_{s-1}(i, j, k)-C_{s}(i, j, k)\right)$ is the signal difference which contains the information between these two scales and is the discrete set of $128^{3}$ wavelet coefficients $W_{s}(i, j, k)$ associated with the wavelet transform by $\Psi(x, y, z)$ (Fig. 1$)$ : $W_{s}(i, j, k)=C_{s-1}(i, j, k)-C_{s}(i, j, k)$.

\subsection{Extracting significant coefficients}

In order to remove non-zero wavelet coefficients generated by noise fluctuations in the $3 \mathrm{D}$ distributions, a local thresholding is applied at each scale in the space of wavelet coefficients. Thresholds are set at each scale and each position by estimating the noise level generated at the same scale by a uniform random signal built with the same gross-characteristics as the observed one at the position considered.

- Local hard-thresholding procedure.

The noise $N_{s}(i, j, k)$ is estimated at the position of each pixel $(i, j, k)$ from the observed distribution in a filter area corresponding to scale $s$. The signal estimation $S_{s}(i, j, k)$, in this area, is:

$S_{s}(i, j, k)=\sum_{l=i-2^{s}}^{i+2^{s}} \sum_{m=j-2^{s}}^{j+2^{s}} \sum_{n=k-2^{s}}^{k+2^{s}} F(l, m, n)$

the noise estimation is:

$$
N_{s}(i, j, k)=\sqrt{\frac{S_{s}(i, j, k)}{\left(2^{s+1}\right)^{3}}} .
$$

At scale $s$, the number of pixels included in the convolution filter volume is $\left(2^{s+1}\right)^{3}$.

The local threshold $\lambda_{s}^{+}(i, j, k)$ is inferred from the local noise $N_{s}(i, j, k)$ through calibration curves established by numerical simulations (see Sect. 2.3) which link it to the noise $N_{s}(i, j, k)$. Then the hard-thresholding on wavelet coefficients follows:

$$
W_{s}(i, j, k)= \begin{cases}W_{s}(i, j, k) & \text { if } W_{s}(i, j, k) \geq \lambda_{s}^{+}(i, j, k) . \\ 0 & \text { otherwise }\end{cases}
$$

- Segmentation procedure.

A segmentation procedure is applied on thresholded coefficients to localize significant over-densities. This segmentation algorithm detects all maxima in a moving box of filter size volume. Neighbouring pixels are aggregated around each maximum until the intensity of wavelet coefficient stop decreasing. This is the classical method of valley lines. All aggregated coefficients around a maximum are considered to sign one structure. All structures are labelled and defined pixel by pixel. This procedure has the advantage of splitting coalescent structures.

\subsection{Thresholding calibration}

- Choice of significant probability.

Following Lega et al. (1996), thresholds $\lambda$ are defined with respect to the probability $P$ that the coefficient $W_{s}(i, j, k)$ is larger than $\lambda_{s}^{+}$(or smaller than $\lambda_{s}^{-}$) at a given scale $s$ in the distribution of positive coefficients (or negative coefficients).

The probability is

$P\left(W_{s}(i, j, k) \geq \lambda_{s}^{+}\right)=\frac{\int_{W_{s}=\lambda_{s}^{+}}^{+\infty} f\left(W_{s}\right) \mathrm{d} W_{s}}{\int_{W_{s}=0}^{+\infty} f\left(W_{s}\right) \mathrm{d} W_{s}}=\epsilon$

where $f$ is the frequency of positive wavelet coefficients at scale $s$. $P$ was set to achieve the best compromise between false detections and losses at each scale with a set of uniform background simulations containing gaussian structures of different sizes and signal to noise ratio. This optimum was found between $510^{-4}$ and $10^{-4}$ in all experiments and in all scales under reasonable signal to noise ratio. Then $\epsilon=10^{-4}$ was fixed for the rest of the calibration.

- Calibration curves.

A calibration curve is obtained for each scale with an other set of different Poisson noise simulations. Noises $N_{s}(i, j, k)$ are estimated at each pixel $(i, j, k)$ from the observed distribution in the same way as described in Sect. 2.2. The mean $\overline{N_{s}}$ of Poisson noise distribution obtained is linked with threshold $\lambda_{s}^{+}\left(\right.$or $\lambda_{s}^{-}$) defined from $P$ giving the calibration curve for scale $s$.

\section{Palliative age determination method}

We succeed in estimating individual ages from Strömgren photometry (Figueras et al. 1991; Masana 1994; Asiain et al. 1997) for a third of the sample (see Paper II). To rule out any bias coming from an analysis of this sub-sample alone, we propose an empirical palliative age estimation method based on the Hipparcos absolute magnitude and colour for the rest of the sample. On a first step, we use existing ages to draw a plot of ages versus $\left(M_{v},(B-V)\right)$. A primary age parameter is assigned as the mean age associated to a given range of $\left(M_{v},(B-V)\right)$ (Sect. 3.1). Then Strömgren age data are used a second time to assign a probability distribution of palliative ages as a function of the primary age parameter (Sect. 3.2). 


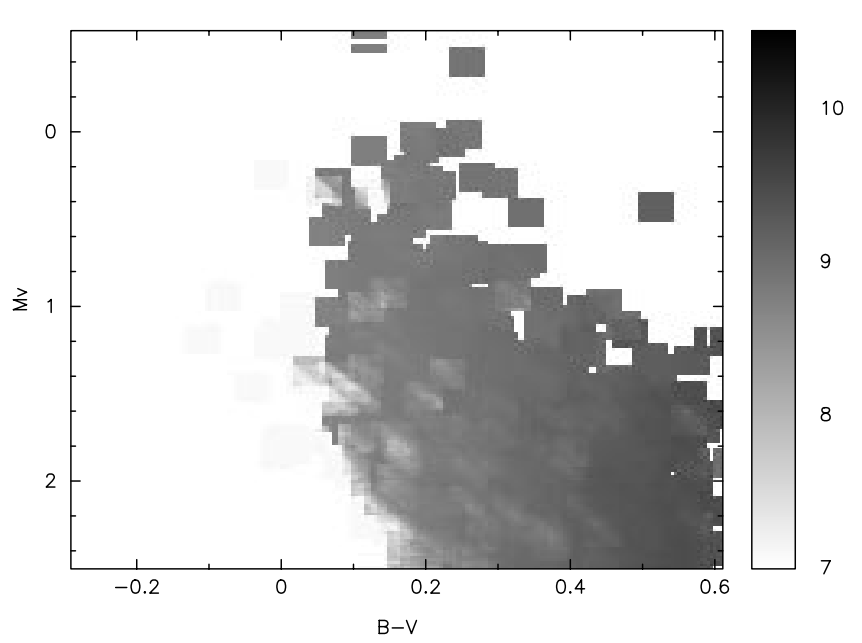

Fig. 2. Primary age parameter versus $\left(M_{v},(B-V)\right)$ relationship calibrated with the Strömgren sub-sample. Grey levels represent the $\log ($ age $)$ value

\subsection{Primary age parameter}

Strömgren ages are available for 1077 A-F type stars with spectral types later than A3 $(B-V \geq 0.08)$ because the metallicity cannot be determined for A0-A3 spectral types. With this sub-sample a relation between the absolute magnitude $M_{v}$, the colour indice $B-V$ and the age has been calibrated on a grid to extrapolate a less accurate age for the rest of our sample (1900 stars). Since the relation cannot be calibrated with Strömgren ages for spectral types between A0 and A3, we add 30 known very young stars $\left(\sim 10^{7} \mathrm{yr}\right)$ belonging to the CentaurusCrux association (see Sect. 4 ) which have $B-V \leq 0.08$. Consequences on the inferred age distribution are investigated in Sect. 3.2. Available Strömgren ages are averaged on a $200 \times 200$ grid, ranging from $[-0.5,2.5]$ in absolute magnitude and $[-0.1,0.6]$ in $B-V$. The result is smoothed and extrapolated to empty cells, where feasible, using a $10 \times 10$ moving window. This process produces a primary age parameter (Fig. 2). In Fig. 3, Strömgren age indicators are plotted against this primary age parameter. Obviously, there is not a one to one relation between the primary age parameter and the Strömgren age. Very young Strömgren ages are the most affected by the degeneracy of this relation; while the bulk of primary age parameters older than $\log ($ age $)=8.7$ are in relatively good agreement with Strömgren ages. Only 14 among the 1900 stars have $\left(M_{v},(B-V)\right)$ out of the calibrated grid and do not have primary age parameter. On the basis of Fig. 3, we can assign a probability distribution for the Strömgren age to each primary age parameter.

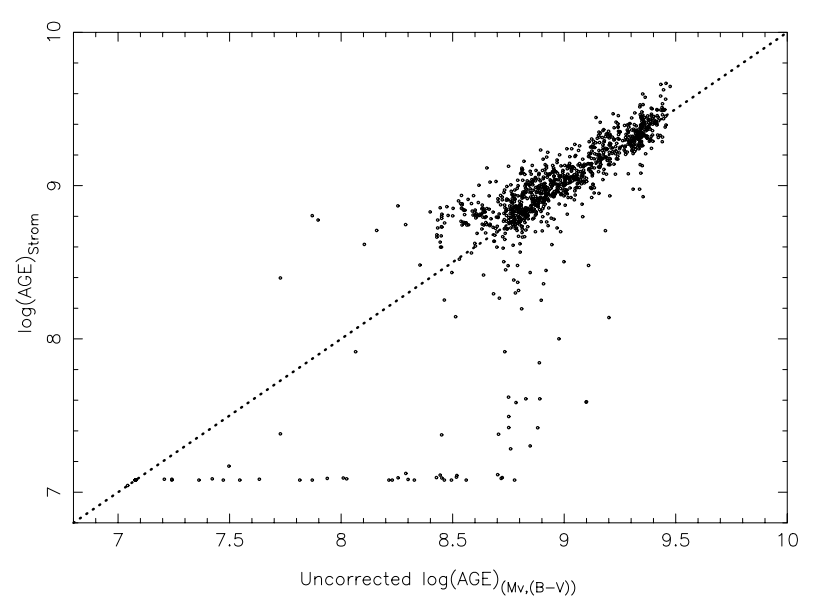

Fig. 3. Primary age parameter versus Strömgren age

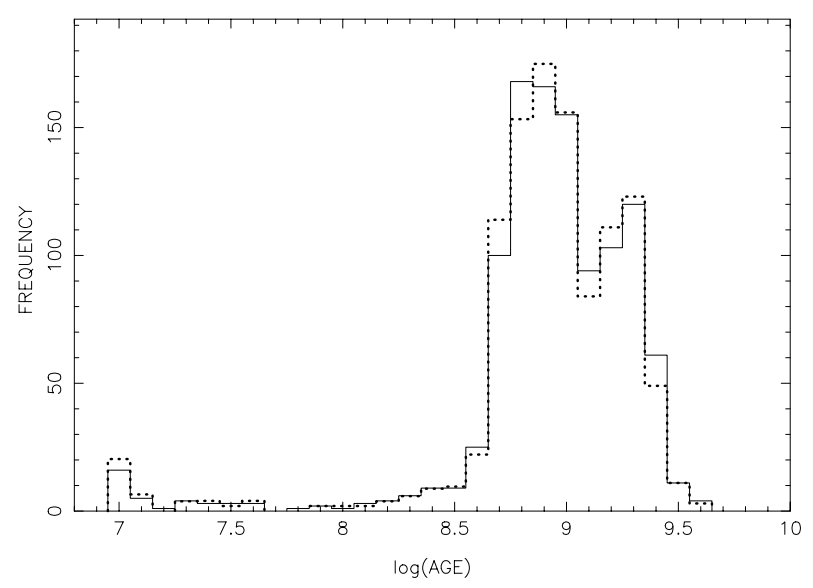

Fig. 4. Strömgren age distribution obtained with 1077 stars (full line) and palliative age distribution for the same sub-sample (dotted line)

\subsection{Palliative ages}

Given a primary age parameter $i$, Fig. 3 can be read as giving a discrete probability distribution $P(j / i)$ of Strömgren ages $j$ under $i . P(j / i)$ is given by:

$P(j / i)=\frac{n(i / j)}{q(i)}$

where $n(i, j)$ is the number of stars in cell $(i, j)$ and

$q(i)=\sum_{j} n(i, j)$

These probabilities are discrete on a grid with an adaptive bin size to keep at least 3 stars per bin. This process produces a palliative age distribution which is free from possible biases affecting the sub-sample of stars with Strömgren photometry for stars with $B-V \geq 0.08$.

The small deviations from the distribution of original Strömgren ages (Fig. 4) are due to finite bin steps used in the discretisation process. The palliative age distribution of the sub-sample without Strömgren photometry (Fig. 5) 


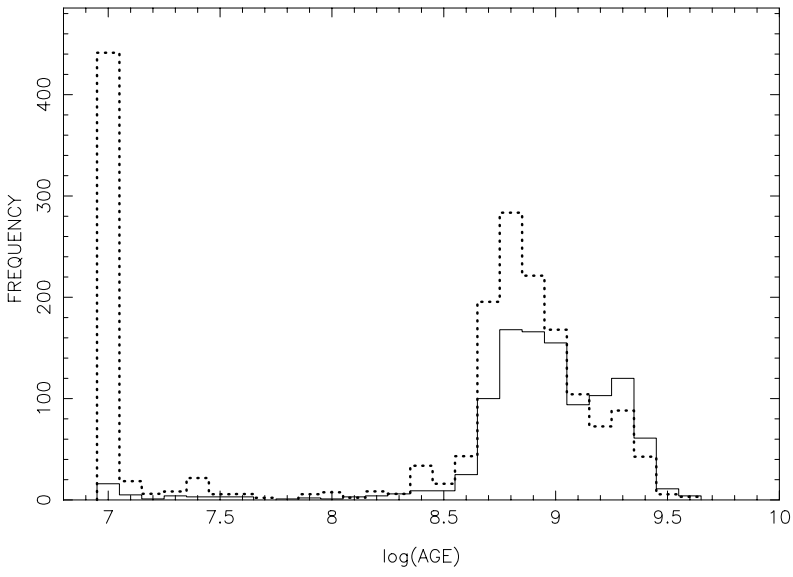

Fig. 5. Strömgren age distribution obtained with 1077 stars (full line) and palliative age distribution for the remainder, i.e. stars without Strömgren photometry (dotted line)

shows a great difference for very young ages with respect to the Strömgren age distribution. The great peak at $\sim 10^{7}$ yr partly results from the poor calibration of the relation between age and $\left(M_{v},(B-V)\right)$ for $B-V \leq 0.08$. If the calibration of the relation is realized without the additional Centaurus-Crux stars, we would obtain a frequency, at $\sim 10^{7} \mathrm{yr}$, of $\sim 170$ stars instead of $\sim 440$ stars. It means that there are at least 170 stars of few $10^{7}$ year old and that the difference of $\sim 270$ stars is composed of A0-A3 type stars which may be older. How older are they? Using theoretical isochrones from Bertelli et al. (1994), a relation between the total lifetime of a star at the turnoff point and the color indice $(B-V)$ can be infered. We can show that the total lifetimes of A0-A3 type stars are between $4-610^{8} \mathrm{yr}$, if the metallicity of the stars are between $Z=0.008$ and $Z=0.02$. Taking the half lifetime of the star as the most probable age, we obtained, for these 270 stars, an age distribution in the range $2-310^{8} \mathrm{yr}$ $(\log ($ age $)=8.3-8.5)$. This distribution of these half lifetimes remains very well separated from the older stars in the sample. To summarize, the great peak at $10^{7} \mathrm{yr}$ contains at least 170 stars of $\sim 10^{7} \mathrm{yr}$ and 270 stars for which ages may spread up to $310^{8}$ yr. These stars belong to the younger part of the sample.

The palliative ages are statistical ages. Hence, they sometimes produce artifacts or young ghost peaks in some stream age distributions. Such dummy young peaks will always appear as the weak counterpart of a heavy peak around $\log ($ age $)=8.7$. Nevertheless palliative ages permit to shed light on the age content of the phase space structures when Strömgren data are sparse.

\section{Clustering}

\subsection{Searching for clusters}

$(X, Y, Z)$ distributions range from -125 pc to +125 pc and are binned in a Sun centered orthonormal frame, $X$-axis towards the galactic center, $Y$-axis in the rotation direction and $Z$-axis towards the north galactic pole. The discrete wavelet analysis is performed on five scales: 9.7, $13.6,21.5,37.1$ and $68.3 \mathrm{pc}$. These values correspond to the size of the dilated filter $h$ at each scale.

Stars belonging to each volume defined by significant coefficients are collected. Due to the over-sampling of the signal by the "à trou" algorithm, some structures are detected on several scales. A cross-correlation has been done between all scales to keep the structure at the largest scale provided that there is no sub-structure at a lower scale (higher resolution). We control a posteriori the efficiency of the detection by calculating for each over-density the probability to find an identical concentration from the total volume of the sample in a uniform random population (see probability $P_{2}$ in Table 1 ). The method also succeeds in finding over-densities with a high probability to be generated by a poissonian process. An iterative 2.5 sigma clipping procedure is done on tangential velocity distributions for each group to remove field stars and select structures with coherent kinematics (Table 1 and Figs. 6, 7, 8, 9 and 10).

\subsection{Detailed cluster characteristics}

The volumes selected by the segmentation procedure content 10 per cent of the stars. After the 2.5 sigma clipping procedure on the tangential velocities, only 7 per cent are still in clusters or groups. Most of them are well known: Hyades, Coma Berenices, Ursa Major open clusters (hereafter $\mathrm{OCl}$ ) and the Scorpio-Centaurus association. The following cases are the most interesting either because of newly discovered features associated to well known structures (Scorpio-Centaurus association) or simply because they were undetected up to now (Bootes and Pegasus 1 and 2).

1. The Scorpio-Centaurus association shows three precisely limited over-densities with an elongated shape crossing the galactic disc (see scale 4 on Paper II, Fig. 5): lower Cen-taurus-Crux, upper CentaurusLupus which both have identical proper motions and a third unknown clump at $\left(l=325.4^{\circ}, b=-34.4^{\circ}\right)$ with slightly different proper motions along $l$ axis (see cluster 9 in Table 1). We do not find previous mention of this extension of the Scorpio-Centaurus. Unfortunately very few of these stars have Strömgren photometry. In the case of Centaurus-Crux (see Figs. 6), among 
Table 1. Main characteristics of detected spatial structures after iterative 2.5 sigma clipping procedure on tangential velocities: galactic coordinates $(l, b)$, distance $D$, mean tangential velocities $\left(\overline{T_{1}}, \overline{T_{\mathrm{b}}}\right)$, tangential velocity dispersions $\left(\sigma_{T_{1}}, \sigma_{T_{\mathrm{b}}}\right)$, number of selected stars $N_{\text {sel }}$. Other parameters are given: scales of detection $S$, volume $v$ occupied by the total over-density, number of expected stars $N_{\mathrm{e}}$ in this volume according to the mean density, number of observed stars $N_{\mathrm{o}}$ before selection on tangential velocities, probability $P_{1}$ to find such a concentration from a poissonian distribution in this $v$ volume and probability $P_{2}$ to find this volume $v$ with this concentration within the $125 \mathrm{pc}$ radius sphere. A value of .001 indicates a probability $P \leq 0.001$

\begin{tabular}{|c|c|c|c|c|c|c|c|c|c|c|c|c|c|c|}
\hline Structure & $\begin{array}{r}l \\
(\operatorname{deg})\end{array}$ & $\begin{array}{l}b \\
(\operatorname{deg})\end{array}$ & $\begin{array}{r}D \\
(\mathrm{pc})\end{array}$ & $\overline{T_{1}}$ & $\begin{array}{r}\sigma_{T_{1}} \\
(\mathrm{kn}\end{array}$ & $\begin{array}{r}\overline{T_{\mathrm{b}}} \\
\left.\mathrm{s}^{-1}\right)\end{array}$ & $\sigma_{T_{\mathrm{b}}}$ & $N_{\text {sel }}$ & $S$ & $\begin{array}{r}v \\
\left(\mathrm{pc}^{3}\right)\end{array}$ & $N_{\mathrm{e}}$ & $N_{\mathrm{o}}$ & $P_{1}$ & $P_{2}$ \\
\hline 1 & 227.65 & -6.14 & 117 & \multicolumn{4}{|c|}{ Possible double system } & 2 & 2 & 831 & 0.302 & 2 & .037 & .999 \\
\hline 2 & 58.52 & +35.89 & 54 & -4.9 & 0.6 & 10.1 & 6.8 & 2 & 2 & 20 & 0.007 & 2 & .001 & .999 \\
\hline 3 & 83.96 & -42.34 & 81 & \multicolumn{4}{|c|}{ Possible double system } & 2 & 2 & 5 & 0.002 & 2 & .001 & .933 \\
\hline 4 & 40.71 & -0.60 & 121 & 6.0 & 3.3 & -5.5 & 5.4 & 4 & 2 & 696 & 0.253 & 4 & .001 & .807 \\
\hline 5 & 231.58 & -20.34 & 101 & \multicolumn{4}{|c|}{ Possible double system } & 2 & 2 & 77 & 0.02 & 2 & .001 & .999 \\
\hline $6 \mathrm{U} \mathrm{Ma}$ & 128.44 & +59.97 & 25 & -12.5 & 1.7 & 1.5 & 2.8 & 6 & $2-3$ & 1191 & 0.43 & 6 & .001 & .042 \\
\hline 7 Cen-Crux & 303.91 & +2.95 & 105 & -17.9 & 3.9 & -7.4 & 2.1 .33 & $2-4$ & 52229 & 19.0 & 36 & .001 & .050 & \\
\hline 8 & 228.98 & +28.84 & 110 & -7.6 & 11.4 & -17.0 & 8.3 & 8 & 4 & 16093 & 5.8 & 8 & .236 & .999 \\
\hline 9 & 325.40 & -34.41 & 111 & -20.9 & 13.9 & -8.1 & 8.5 & 6 & 4 & 11718 & 4.2 & 6 & .257 & .999 \\
\hline 10 Cen-Lup & 335.27 & +17.30 & 117 & -17.7 & 10.5 & -5.2 & 10.5 & 9 & 4 & 33028 & 12.0 & 12 & .540 & .999 \\
\hline 11 Coma & 214.50 & +83.6 & 88 & 2.9 & 1.8 & -6.4 & 2.3 & 16 & $2-4$ & 10968 & 3.9 & 21 & .001 & .001 \\
\hline 12 Hyades & 180.26 & -21.38 & 47 & 19.4 & 2.8 & 14.2 & 2.1 & 22 & $2-4$ & 63986 & 23.3 & 45 & .001 & .005 \\
\hline 13 & 34.39 & +33.89 & 108 & -9.5 & 7.6 & -0.5 & 14.3 & 7 & 4 & 18316 & 6.6 & 7 & .499 & .999 \\
\hline 14 Bootes & 97.64 & +56.68 & 105 & 5.0 & 1.6 & 6.8 & 1.3 & 5 & $2-4$ & 12574 & 5.9 & 13 & .001 & .455 \\
\hline 15 Pegasus 1 & 60.77 & -32.57 & 97 & 0.6 & 3.5 & -12.0 & 3.9 & 10 & $4-5$ & 71687 & 26.0 & 35 & .054 & .998 \\
\hline Pegasus 2 & 54.20 & -31.33 & 89 & 14.2 & 2.8 & -21.2 & 5.2 & 8 & $4-5$ & $"$ & $"$ & $"$ & $"$ & $"$ \\
\hline 16 & 80.44 & +32.61 & 109 & 4.7 & 16.3 & 0.3 & 3.7 & 8 & 4 & 17651 & 6.4 & 8 & .316 & .999 \\
\hline 17 Hyades' tail & 163.56 & -8.34 & 67 & 20.8 & 8.5 & -2.6 & 4.4 & 39 & 5 & 246202 & 89.5 & 90 & .496 & .999 \\
\hline Total & & & & & & & & 189 & & & & 299 & & \\
\hline Percentage & & & & & & & & $\mathbf{7} \%$ & & & & $10 \%$ & & \\
\hline
\end{tabular}

33 stars, 3 stars having a Strömgren age are rather old. One of these stars is flagged as a double system in the Hipparcos catalogue, which could alter the Strömgren photometry. The two others probably do not belong to the association but are not rejected by the selection on tangential velocities because of proper motions close to the association ones. However this association is known to be an O-B association, which means that it is younger than a few tens of million years.

The palliative age distributions show that all the three clumps are composed by the youngest stars of the sample. The analysis of the velocity field (see Sect. 5.3.1) reveals that these density inhomogeneities are embedded in a stream-like structure moving through all the 125 pc sphere.

Two new structures are found both probably on their way towards disruption. In the following these loose clusters are designated after the constellation they are found in.

2. Bootes (Fig. 8) is composed of only 5 stars at a mean distance of $105 \mathrm{pc}$ and centred on $\left(l=97.6^{\circ}, b=\right.$ $\left.+56.6^{\circ}\right)$. This small clump is interesting for several reasons. It is located at the same $x$ and $z$ coordi- nates as Coma Ber. OCl (Fig. 7) but 62 pc ahead along $y$-axis with exactly the same space velocity components $(U, V, W)=(-3.1,-7.8,-0.8) \mathrm{km} \mathrm{s}^{-1}$. These coincidences suggest a common origin for these two structures. But their respective age distributions (Figs. 7 and 8) show that Bootes is probably younger (peak at $10^{7} \mathrm{yr}$ for the palliative age distribution) than Coma Ber. OCl which is $\sim 610^{8} \mathrm{yr}$ old. Moreover, the smaller tangential velocity dispersions of Bootes tends to confirm this hypothesis. A more detailed investigation among other spectral types is necessary to better define Bootes in velocity space as well as in age content.

3. Pegasus appears to be composed of two different velocity components (Figs. 9, 10). This feature do not permit to extract coherent velocity structures by means of the sigma clipping procedure. A hand selection was realized on the basis of the whole tangential velocity distribution. Pegasus 1 with 10 stars and Pegasus 2 with 8 stars are obtained with mean distances of respectively 97 and $89 \mathrm{pc}$ and centred on $\left(l=60.7^{\circ},-32.5^{\circ}\right)$ and $\left(54.2^{\circ},-31.3^{\circ}\right)$. Their age distributions are not very precise because of the lack of Strömgren ages. However, it seems that 

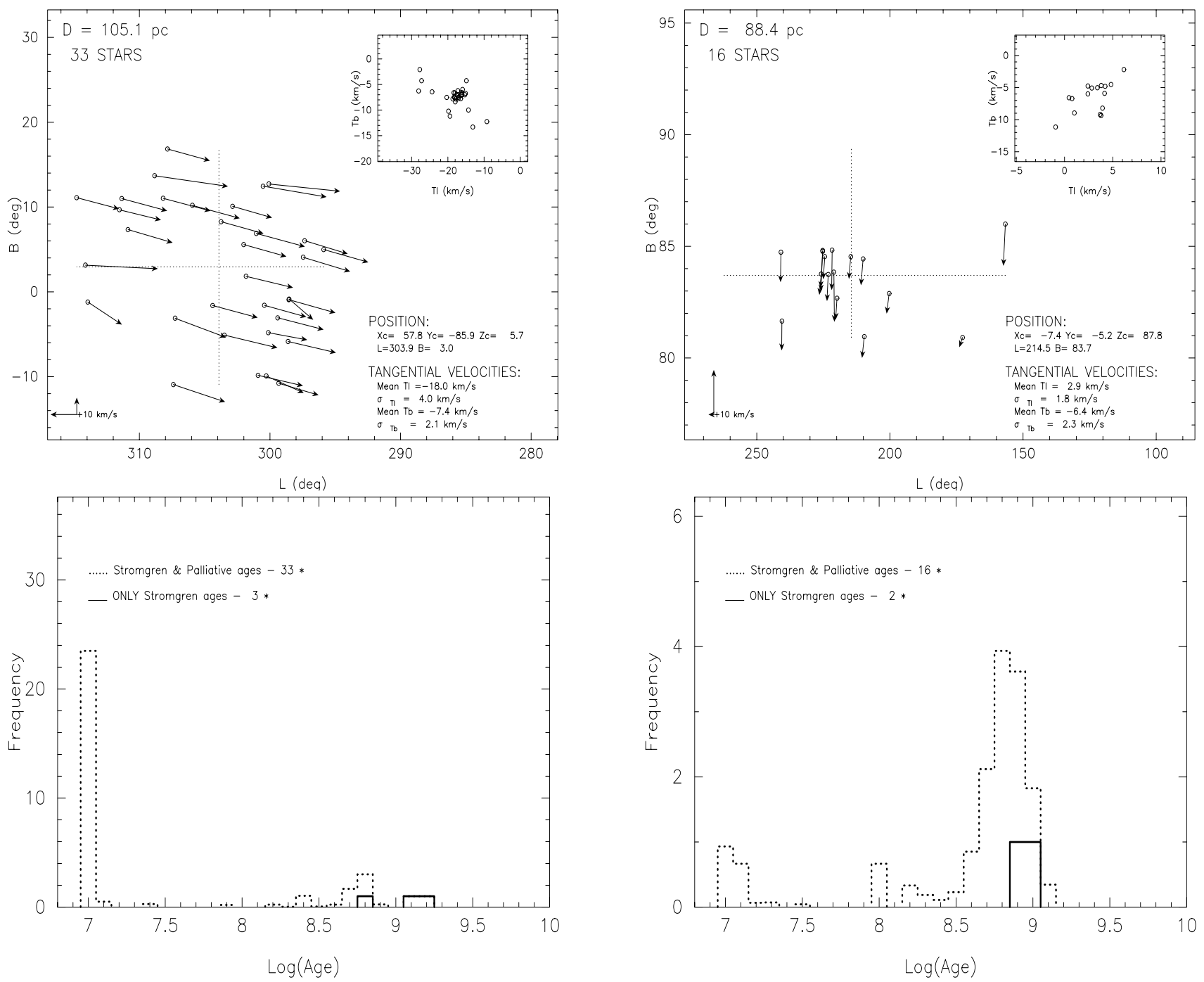

Fig. 6. Lower Centaurus-Crux after selection on tangential velocities (top) and its age distribution (bottom)

Pegasus 1 has an age peak at $810^{8} \mathrm{yr}$. It has no very young ages but 3 Strömgren ages are between $810^{8}$ and $1.210^{9}$ yr which would mean an age older than Pegasus 1. This complex structure gives an instantaneous snapshot of the interactions affecting groups, clusters or associations: merging-like process can take part in dissolving spatial inhomogeneities.

\section{Streaming}

The sub-sample with observed radial velocities is incomplete and contains biases since the stars are not observed at random. To keep the benefits of the sample completeness, a statistical convergent point method is developed to analyze all the stars (Sect. 5.1). However, this method creates spurious members among detected streams with

Fig. 7. Coma Berenices open cluster after selection on tangential velocities (top) and its age distribution (bottom)

wavelet analysis, Sect. 5.2.1 describe the procedure to handle their proportion. Moreover the fraction of field stars detected as stream members is also evaluated through the procedure in Sect. 5.2.2.

\subsection{Recovering 3-D velocities from proper motions: $A$ convergent point method}

$U, V$ and $W$ velocities are reconstructed from Hipparcos tangential velocities by a convergent point method for stars which belong to streams. All pairs of stars are considered; each pair gives a possible convergent point (assuming that both stars move exactly parallel) and the radial velocity is inferred for each component. Then fully reconstructed velocities of the two stars, $V_{1}$ and $V_{2}$, are considered only if $\left|V_{1}-V_{2}\right|$ does not exceed a fixed selection criterion. This pre-selection of reconstructed velocities eliminates most of false reconstructions. In the 

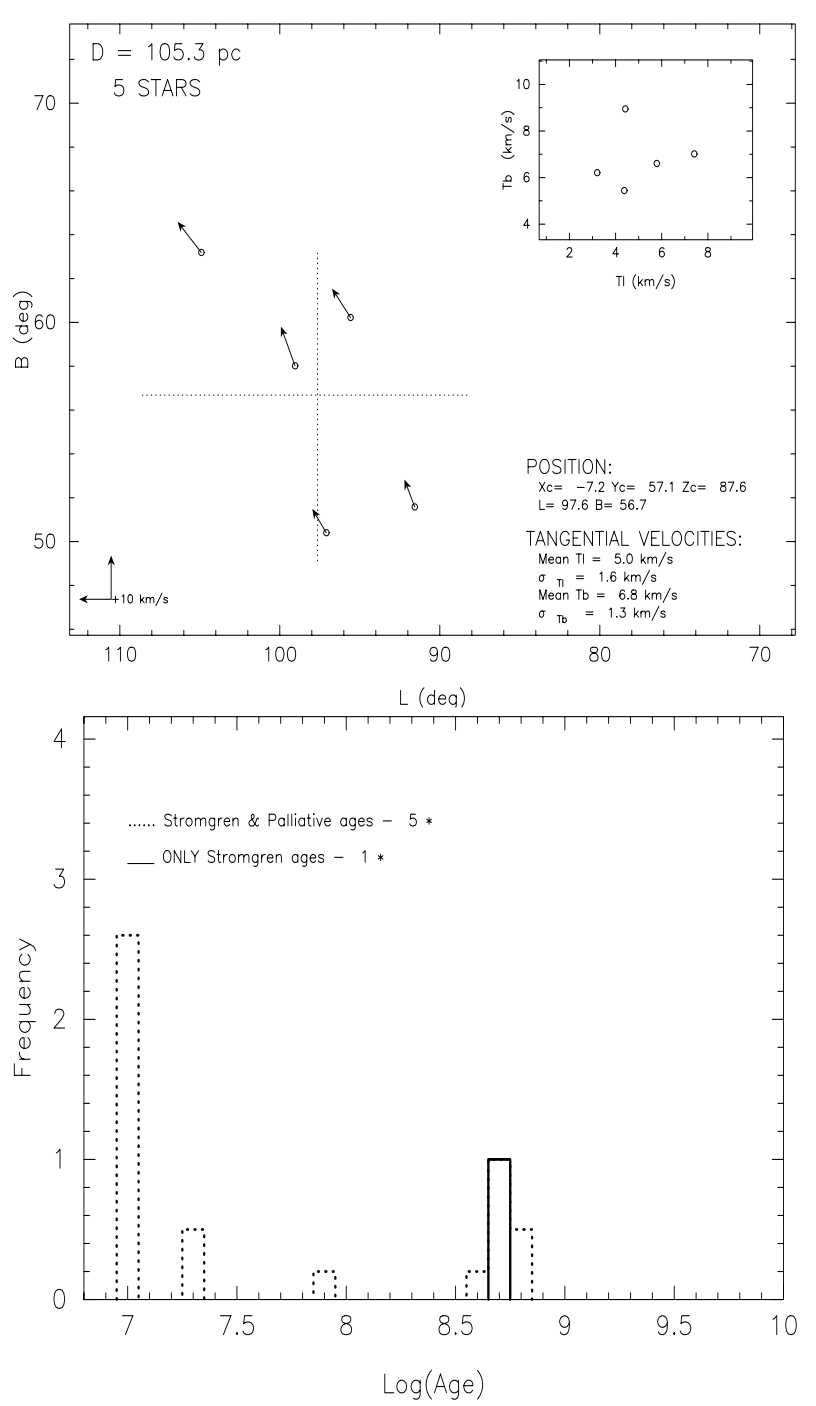

Fig. 8. Bootes loose cluster after selection on tangential velocities (top) and its age distribution (bottom)

following, the criterion is fixed to $0.5 \mathrm{~km} \mathrm{~s}^{-1}$. The convergent point algorithm (see Fig. 11) follows the steps:

1. keep a pair of stars $\left(S_{1}, S 2\right)$,

2. define vectors $\boldsymbol{N}_{\mathbf{1}}$ and $\boldsymbol{N}_{\mathbf{2}}$ which are perpendicular respectively to the plane $\left(\boldsymbol{O S}_{\mathbf{1}}, \boldsymbol{V}_{\mathbf{1} \|}\right)$ and $\left(\boldsymbol{O S}_{\mathbf{2}}, \boldsymbol{V}_{\mathbf{2} \|}\right)$,

3. obtain $\boldsymbol{N}_{\mathbf{1}} \times \boldsymbol{N}_{\mathbf{2}}=\boldsymbol{O A}$ which is the direction of the hypothetical convergent point $\mathrm{A}$,

4. test the coherence of this direction with both tangential velocities: if $\operatorname{sgn}\left(\boldsymbol{O A} \cdot \boldsymbol{V}_{\mathbf{1 \|}}\right) \neq \operatorname{sgn}\left(\boldsymbol{O A} \cdot \boldsymbol{V}_{\mathbf{2} \|}\right)$, it is not a convergent point. Go to 1 ,

5. calculate angles between star directions and the convergent point: $\Phi_{1}=\left(\widehat{\boldsymbol{O}} \widehat{\boldsymbol{S}_{\mathbf{1}}, \boldsymbol{O} \boldsymbol{A}}\right)$ and $\Phi_{2}=\left(\widehat{\boldsymbol{O}} \widehat{\boldsymbol{S}_{\mathbf{2}}, \boldsymbol{O} \boldsymbol{A}}\right)$,

6. infer moduli and signs of radial velocities:

$$
\begin{gathered}
V_{\mathrm{R}}=\frac{V_{\|}}{\tan \Phi} \text { if } \Phi<\frac{\pi}{2} \\
V_{\mathrm{R}}=\frac{V_{\|}}{\tan (\Phi-\pi)} \text { if } \Phi>\frac{\pi}{2} \text { and } \Phi \neq \pi
\end{gathered}
$$
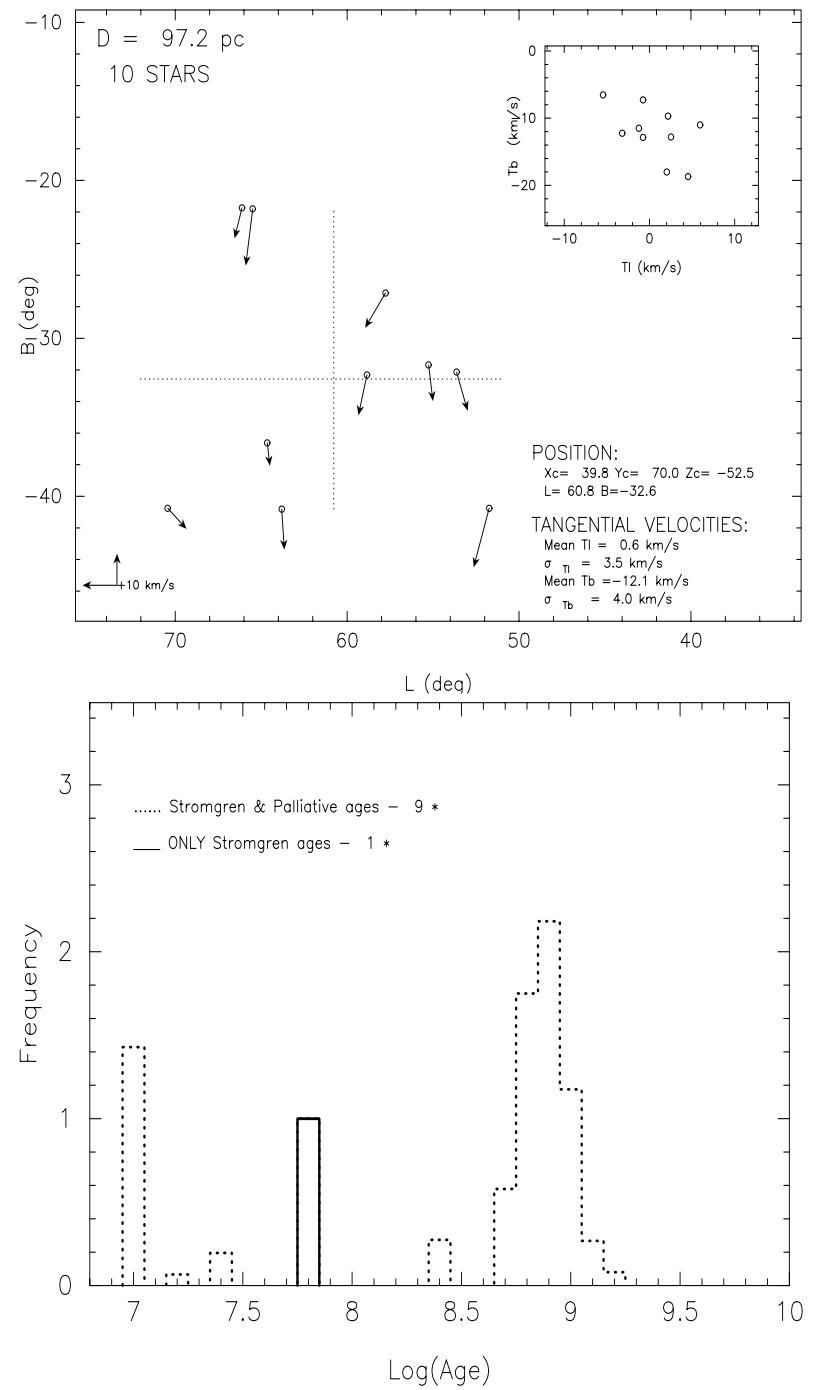

Fig. 9. Pegasus 1 after selection on tangential velocities (top) and its age distribution (bottom)

7. calculate space velocities $V_{1}$ and $V_{2}$ which vectors are strictly parallels by construction

$$
V=\sqrt{V_{\|}^{2}+V_{\mathrm{R}}^{2}}
$$

8. test agreement between $V_{1}$ and $V_{2}$ within tolerance $\eta$ $\left|V_{1}-V_{2}\right| \leq \eta$ with fixed $\eta=0.5 \mathrm{~km} \mathrm{~s}^{-1}$.

Following this process, a large number of may-be velocities are calculated. Several definitions of each star velocity are obtained. All are distributed along a line in the 3D velocity space, part of them being spurious. Real streams produce over-density clumps formed by line intersections. The wavelet analysis detects such clumps in the $(U, V, W)$ distributions. The detection sensitivity is tested numerically by simulating a variety of stream amplitudes and velocity dispersions over a velocity ellipsoid background. Simulations show that our wavelet analysis implementation is able to discriminate streams formed by at least 

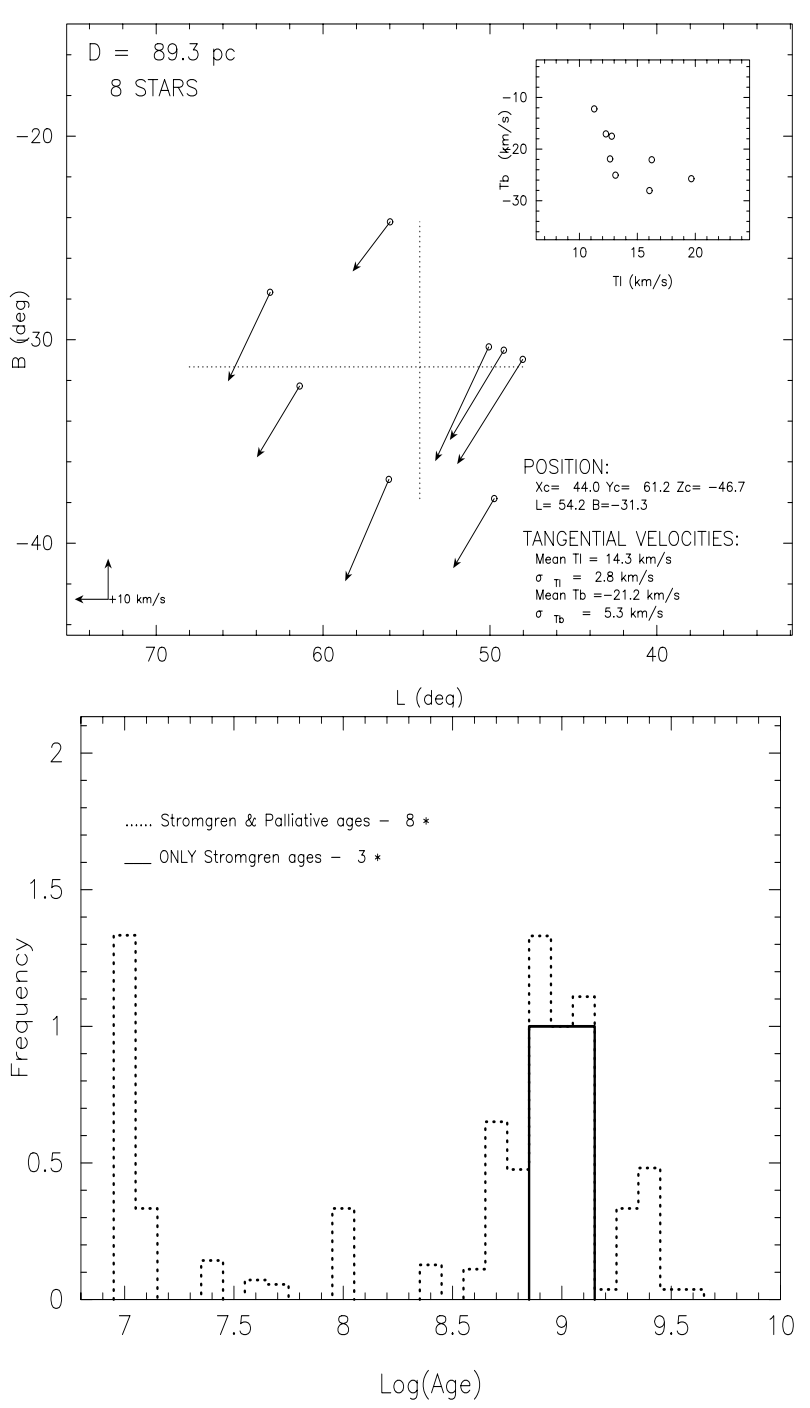

Fig. 10. Pegasus 2 after selection on tangential velocities (top) and its age distribution (bottom)

16 stars non-spatially localized and moving together with a typical velocity dispersion of $2 \mathrm{~km} \mathrm{~s}^{-1}$ in a velocity background matching the sample's one. The scale at which the stream velocity is detected is a measure of the stream velocity dispersion. A more accurate knowledge of this parameter is obtained after the full identification of the members (see Sect. 5.3).

Velocities of open cluster stars are poorly reconstructed by this method because their members are spatially close. For such stars, even a small internal velocity dispersion results a poor determination of the convergent point. For this reason, we have removed stars belonging to the 6 main identified space concentrations (Hyades $\mathrm{OCl}$, Coma Berenices OCl, Ursa Major OCl and Bootes 1, Pegasus 1, Pegasus 2 groups) found in the previous spatial analysis. Eventually, the reconstruction of the velocity field is performed with 2910 stars.

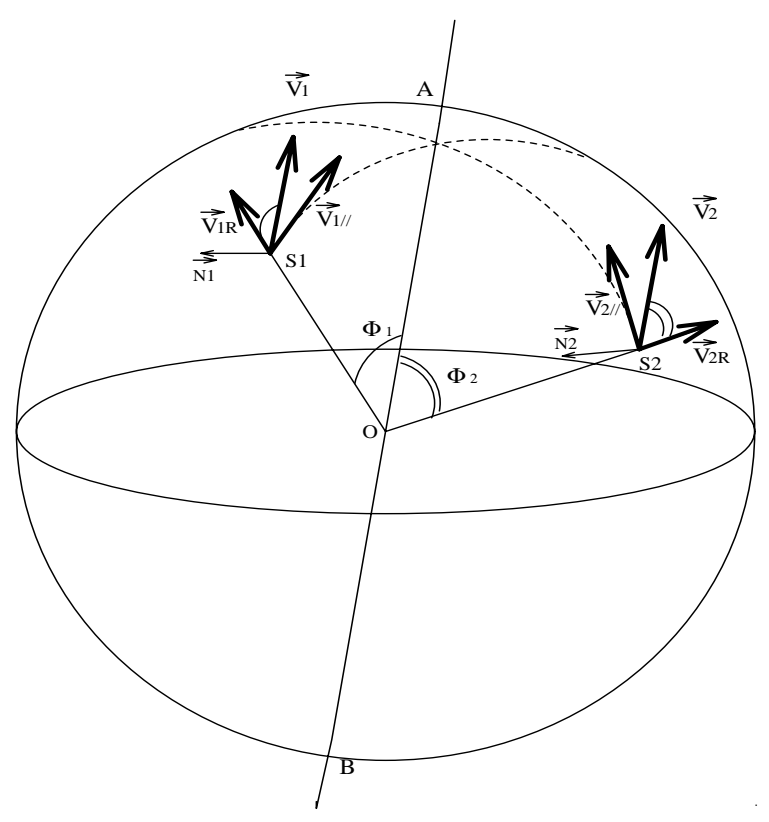

Fig. 11. Implementation of the convergent point method. $\boldsymbol{V}_{\mathbf{1} \|}$, $\boldsymbol{V}_{\mathbf{2} \| \mid}$ are the tangential velocities measured by Hipparcos for two stars. $\boldsymbol{V}_{\mathbf{1} \boldsymbol{R}}, \boldsymbol{V}_{\mathbf{2} \boldsymbol{R}}$, are deduced radial velocities assuming that stars $S_{1}$ and $S_{2}$ belong to the same stream

Reconstructed $(U, V, W)$ distributions are given in an orthonormal frame centred in the Sun velocity in the range $[-50,50] \mathrm{km} \mathrm{s}^{-1}$ on each component. The wavelet analysis is performed on five scales: $3.2,5.5,8.6,14.9$ and $27.3 \mathrm{~km} \mathrm{~s}^{-1}$. In the following, the analysis focuses on the first three scales revealing the stream-like structures because larger ones reach the typical size of the velocity ellipsoid. Once the segmentation procedure is achieved, stars belonging to velocity clumps are identify. The set of velocity definitions of a star may cross two velocity clumps. In this case, the star is associated with the clump in which it appears most frequently.

\subsection{Cleaning stream statistics}

5.2.1. Estimating the fraction of spurious members in velocity clumps

Despite the pre-selection of reconstructed velocities $\left(\left|V_{1}-V_{2}\right| \leq \eta\right)$ some spurious velocities are still present in the field. For this reason, real velocity clumps do include some proportion of spurious members generated by the convergent point method. Estimating the proportion of spurious members in each velocity clump is essential and is done by comparing the mean of reconstructed radial velocities of each star with its observed radial velocity whenever this data is available (1362 among 2910 stars). For each star in a stream, the following procedure is adopted:

1. Only radial velocities reconstructed with other suspected members of the same stream are considered. 


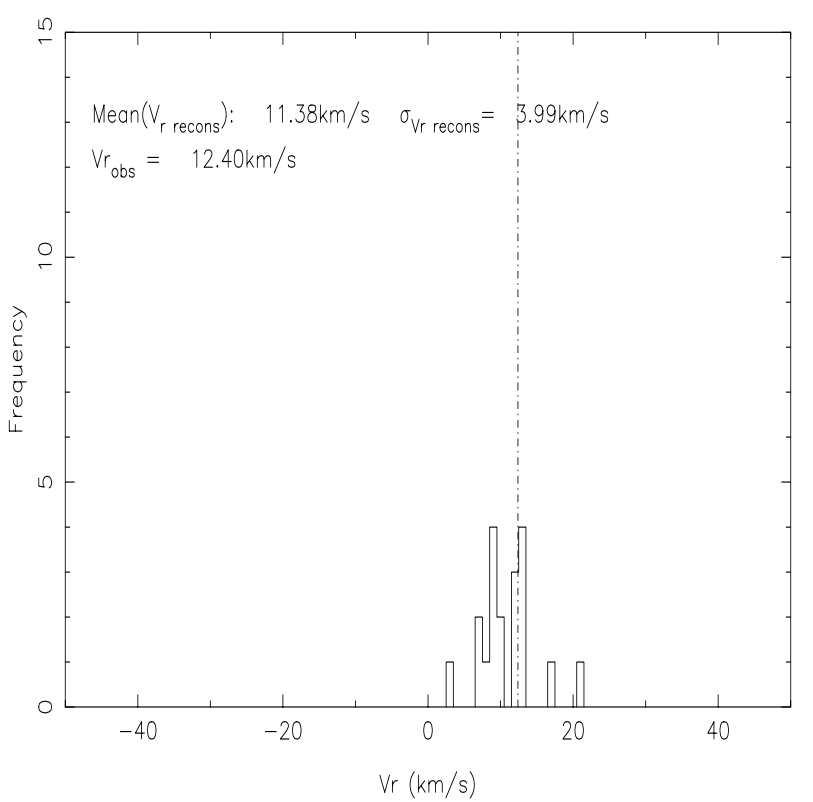

Fig. 12. Example of $V_{R_{\text {rec. }}}$ distribution for one star suspected to be member of a stream and its observed radial velocity (dot dashed line)

2. Mean $\overline{V_{R_{\text {rec. }}}}$ and dispersion $\sigma_{V_{R_{\text {rec. }}} \text { of the reconstructed }}$ radial velocity distribution are calculated (Fig. 12).

3. The star is confirmed to belong to the stream when the normalized residual

$$
R_{N}=\frac{\left|V_{R_{\text {obs. }}}-\bar{V}_{R_{\text {rec. }}}\right|}{\sqrt{\sigma_{V_{R_{\text {rec }}}^{2}}^{2}+\sigma_{V_{R_{\text {obs }}}}^{2}}}
$$

doesn't exceed a value $\kappa$. Residuals take into account errors $\sigma_{V_{R_{\mathrm{obs}}} \text {. }}$ on the observed radial velocities given in the Hipparcos Input Catalogue. These errors are classified into 4 main values: $0.5,1.2,2.5,5 \mathrm{~km} \mathrm{~s}^{-1}$.

The threshold $\kappa$ is fixed empirically on the basis of the normalized residual histogram of all suspected stream members with observed radial velocities (Fig. 13) at scale 2 . It is set to $|\kappa|=3$ in order to keep the bulk of the central peak. The results of this paper are robust to any reasonable change of $|\kappa|$ between 2.5 and 4 .

Noise estimations in each velocity clump for the three first scales (provided that they have at least 3 stars with observed $V_{R}$ ), following the procedure quoted above, are shown in Fig. 14. There are two regimes in these noise estimations: streams with more than 50 initial suspected members $\left(N_{\text {init }}\right)$ which have a contamination by spurious members around 30\%; streams with less than 50 initial suspected members which may have a contamination up to $85 \%$. In this extreme case, should we say that these streams are false detection? Not necessarily, because our denoising method is too drastic towards small streams. Indeed, in those, each star has very few reconstructions of its radial velocity with the other members of the

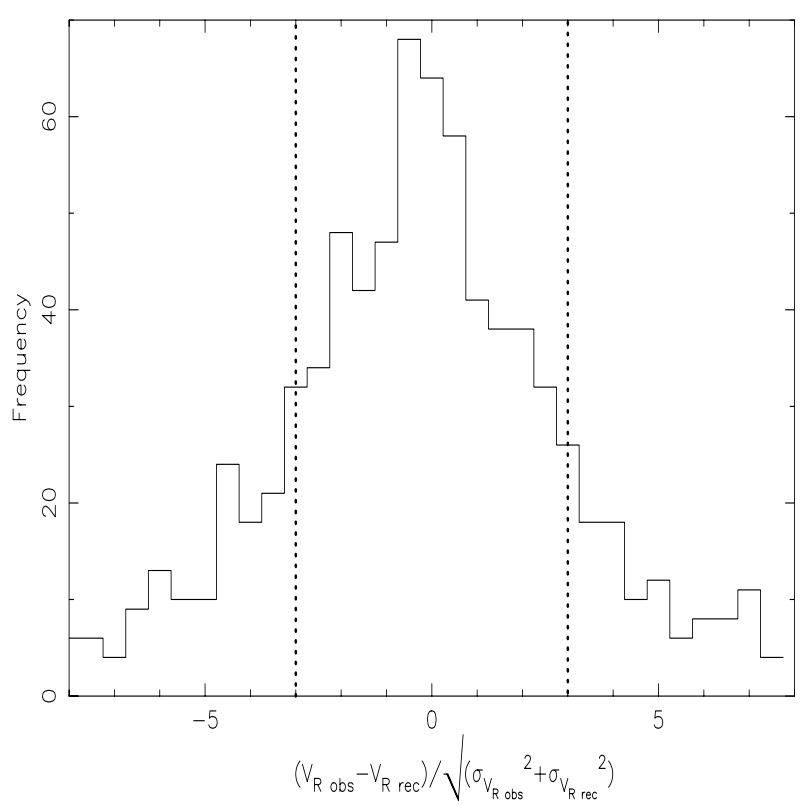

Fig. 13. Distribution of weighted radial velocity deviates (normalized residual) for all suspected stream members with observed radial velocity at scale 2 . Dashed line delimits the area of high probability of false detection

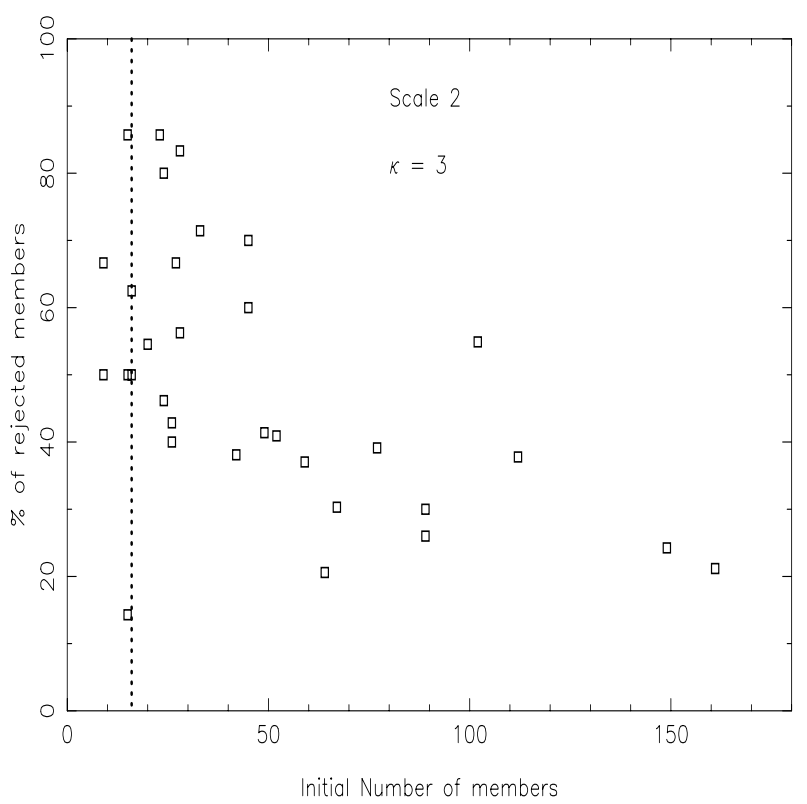

Fig. 14. Proportion of rejected members for all the detected velocity clumps at scale 2 by the selection on observed radial velocity function of the initial number of members. Dashed line indicates the minimum number of stream members requires to detect streams in simulations 
E. Chereul et al.: The distribution of nearby stars in phase space mapped by Hipparcos. III.
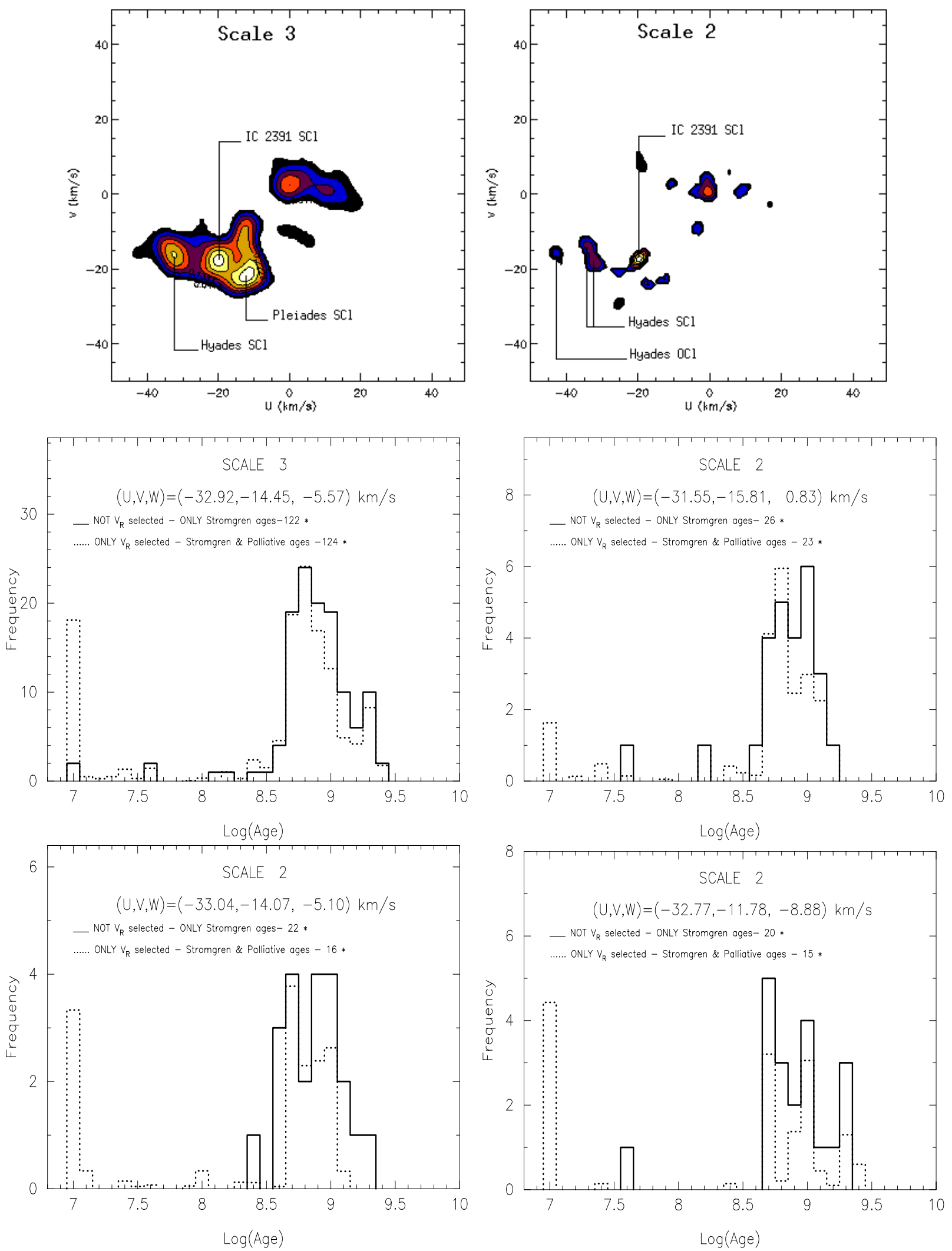

Fig. 15. Hyades Scl. Thresholded wavelet coefficient isocontours at $W=-2.4 \mathrm{~km} \mathrm{~s}^{-1}$ of the velocity field at scale 3 (top left) and scale 2 (top right). This slice in wavelet coefficients at scale 2 reveals two of the three clumps composing the whole supercluster. Age distributions of the whole group (stream 3-10 in Table 2) at third scale (middle left) and of the first sub-stream (stream 2-10 in Table 3) at second scale (middle right). Age distributions of the two last sub-streams (stream 2-18 and 2-25 in Table 3) discovered at scale 2 (bottom) 
same stream. The dispersion of the reconstructed $V_{\mathrm{R}}$ distribution is necessarily small, implying an important normalized residual. Then, the star is often rejected. If we refer to our previous simulations there is a high probability that under 16 initial members, streams are false detection.

At the end of this selection we obtain the number of confirmed members among stars with observed $V_{R}$ in each stream for the three first scales (column $N_{\text {sel }}$ in Tables 2 , 3 and 4 ) and their sum (line Total2 in column $N_{\text {sel }}$ ).

5.2.2. Estimating the proportion of field stars in velocity clumps

The fraction of a smooth distribution filling the velocity ellipsoid of our complete sample, expected inside the velocity volume spanned by the 6 superclusters described bellow, range between $2 \%$ and $4 \%$ depending on the position of the structure with respect to the distribution centroid. Adding up these contributions, $19.2 \%$ of field stars should be expected to fill the total volume occupied by superclusters with pure random coincidence. This is about $20-30 \%$ of the stars detected as supercluster members at scale 3 . However, streams with smaller velocity dispersions (scales 1 and 2) are not significantly affected by this background. Proportions of field stars for the largest structures found at scale 3 are given in 2 at column \% field while it is neglected for the remaining streams.

\subsection{Stream phenomenology}

Tables 2, 3 and 4 give mean velocities, velocity dispersions and numbers of stars remaining after correction of spurious members (procedure 5.2.1) and field stars (procedure 5.2.2) for streams at respectively scale 3,2 and 1. Each stream has $N_{V_{\mathrm{r}}}$ observed radial velocity members. Out of the $N_{V_{\mathrm{r}}}$ stars with radial velocities among suspected stream members, only $N_{\text {sel }}$ get confirmed by procedure 5.2.1. So the ratio $N_{\text {sel }} / N_{V_{\mathrm{r}}}$ is an estimate of the confirmed/suspected ratio in each stream. Applying this ratio to $N_{\text {init }}$ (total stream member candidates) we get the expected number of real stream members in each stream, and the total number of stream members in the sample (Total 3). The percentage of stars in streams in the total sample follows (Total 4). The correction for the uniform background contribution is negligible at scales 1 and 2 ; it is significant at scale 3 where the fraction of stars in streams drops from $63.0 \%$ to $46.4 \%$. In the case of large velocity dispersion structures at scale 3 proportions of field stars is also given in column \% field and the percentage of remaining stream stars is done in column $N_{\text {init }}$ line Total 5.

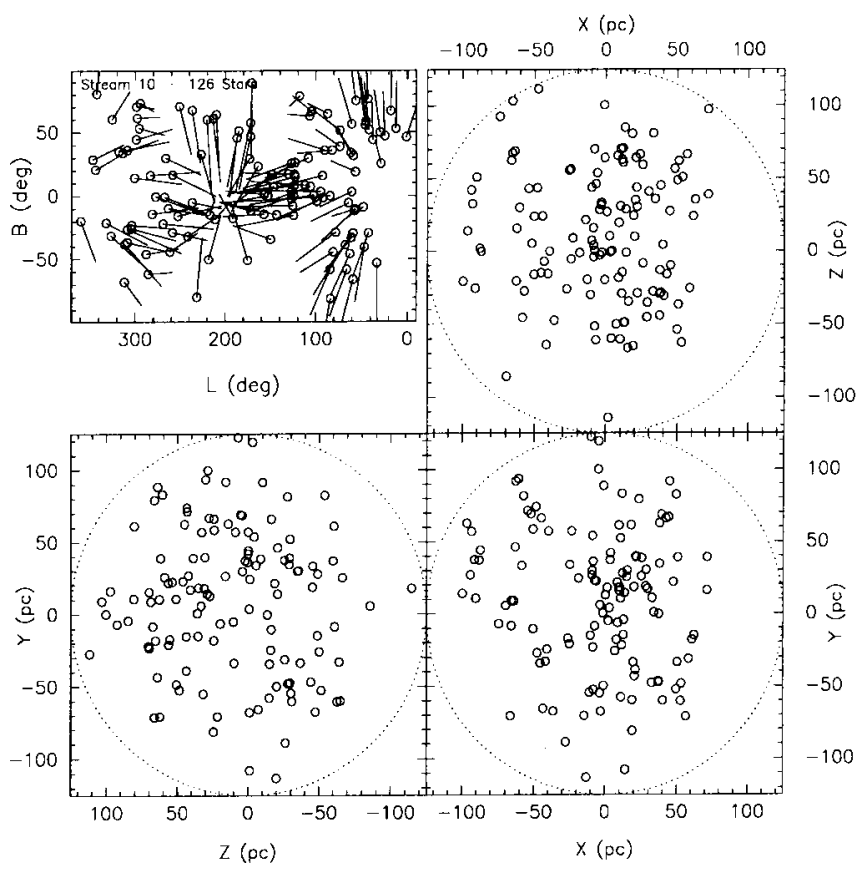

Fig. 16. Space distribution of Hyades $\mathrm{SCl}$ from the $V_{\mathrm{R}}$ selected sub-sample at scale 3 (stream 3-10 in Table 2)

\subsubsection{Particulars on superclusters}

Streams appearing at scale $3\left(\bar{\sigma}_{\text {stream }} \sim 6.3 \mathrm{~km} \mathrm{~s}^{-1}\right)$ correspond to the so-called Eggen superclusters. Four already known such structures are found: the Pleiades, Hyades and Sirius superclusters (hereafter $\mathrm{SCl}$ ) and the whole Centaurus association. Moreover, evidence is given for one additional structure not detected yet. The reason why this supercluster remained undetected is probably the small velocity offset with respect to the Sun's. At smaller scales $\left(\bar{\sigma}_{\text {stream }} \sim 3.8\right.$ and $\left.2.4 \mathrm{~km} \mathrm{~s}^{-1}\right)$ superclusters split into distinct streams of smaller velocity dispersions.

The analysis of the age distribution inside each stream is performed on three different data sets:

- the whole sample (ages are either Strömgren or palliative),

- the sample restricted to stars with Strömgren photometry data (without selection on radial velocity),

- the sample restricted to stars with observed (as opposed to reconstructed) radial velocity data (ages are either Strömgren or palliative).

The selection on photometric ages gives a more accurate description of the stream age content while the last sample permits to obtain a reliable kinematic description since stream members are selected through the 5.2.1 procedure. All mean velocities and velocity dispersions of the streams are calculated with the radial velocity data set. Combining results from these selected data sets generally brings unambiguous conclusions. 
E. Chereul et al.: The distribution of nearby stars in phase space mapped by Hipparcos. III.
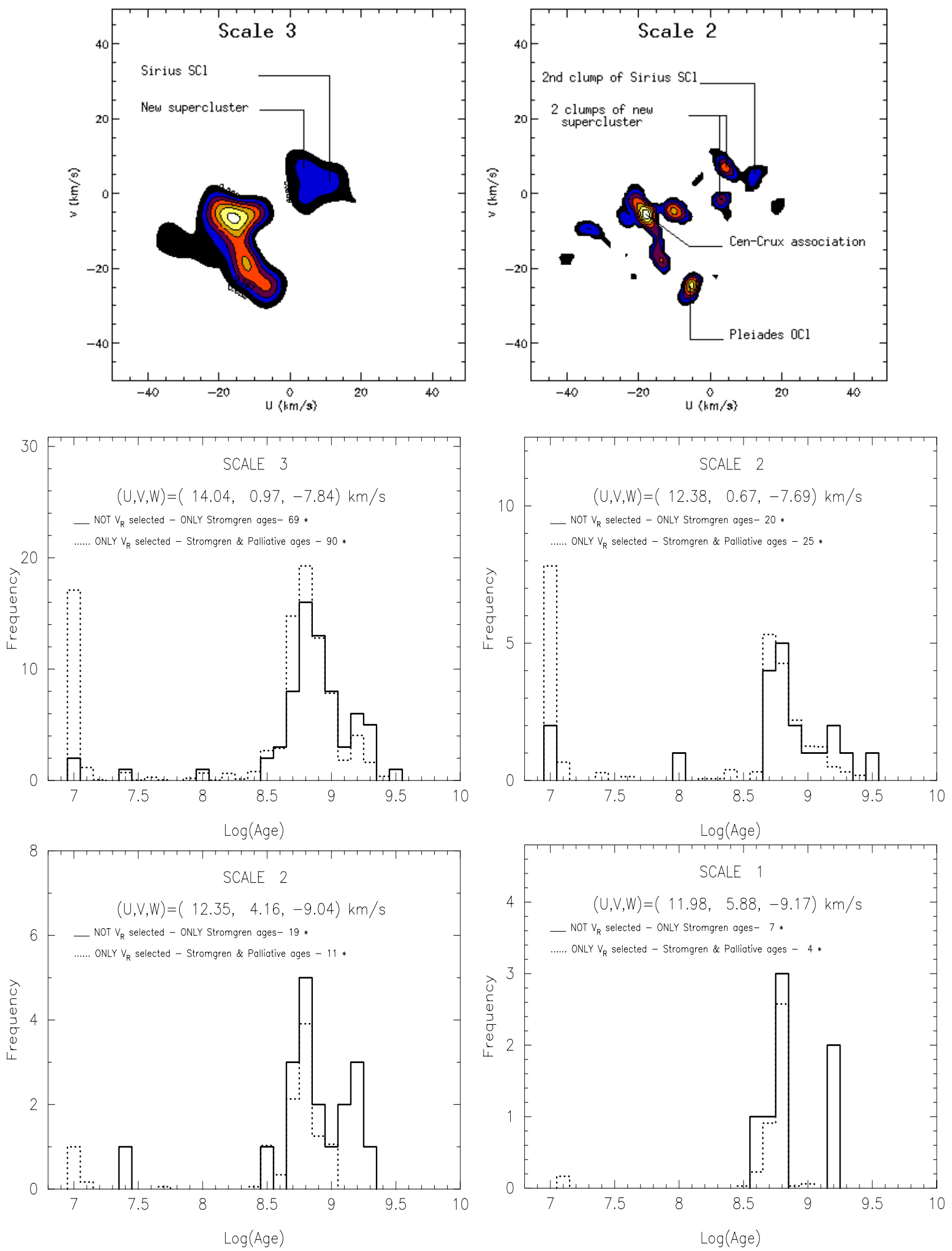

Fig. 17. Sirius Scl. Thresholded wavelet coefficient isocontours at $W=-10.1 \mathrm{~km} \mathrm{~s}^{-1}$ of the velocity field at scale 3 (top left) and scale 2 (top right). At scale 2, Sirius $\mathrm{SCl}$ is composed of 2 main streams (stream 2-37 and 2-41 in Table 3 ). The $2^{\text {nd }}$ is shown on this $W$ slice of wavelet coefficients. Age distributions of the whole Sirius SCl at third scale (middle left) and stream 2-37 at second scale (middle right). Age distribution s of stream 2-41 at scale 2 (bottom left) and stream 1-56 (in Table 4) at scale 1 (bottom right). This latest figure (highest resolution) shows that separating oldest populations is out of reach 
Table 2. Main characteristics of detected streams at scale 3 with filter size of $8.6 \mathrm{~km} \mathrm{~s}^{-1}\left(\bar{\sigma}_{\text {stream }} \sim 6.3 \mathrm{~km} \mathrm{~s}{ }^{-1}\right)$. $\overline{\mathbf{U}} \pm \sigma_{u}, \overline{\mathbf{V}} \pm \sigma_{v}$ and $\overline{\mathbf{W}} \pm \sigma_{w}$ are the mean velocity components and their dispersions calculated, after selection on $V_{\mathrm{R}}$, with the true radial velocity data - $N_{\text {init }}$ is the initial number of stars belonging to the structure - $N_{V_{\mathrm{R}}}$ is the number of stars with observed radial velocity $-N_{\text {sel }}$ is the number of stars among $N_{V_{\mathrm{r}}}$ after selection 5.2.1. The selection has not been done in case where there is less than 3 observed radial velocities. In this last case an d also when none of the stars are selected we do not present the line in the table. However the same stream identifiers are conserved: the first digit is the scale followed by the stream number. Column \% field is the percentage of field st ars estimated in each structure with the procedure 5.2.2. Cross-identification is done with Eggen's superclusters ( $\mathrm{SCl}$ ) and open cluster $(\mathrm{OCl})$ data from Paloùs and Eggen (in Gomez et al. 1990) (see Table 5) Total 1 gives the s um of suspected stream members (Col. $\left.N_{\text {init }}\right)$ and the sum of observed $V_{R}$ among them $\left(\right.$ Col. $\left.N_{V_{\mathrm{R}}}\right)-$ Total 2 gives the sum of all confirmed stream stars among available observed radial velocities (Col. $\left.N_{\text {sel }}\right)$ - Total 3 is the sum of all expected stream members in each stream (Col. $N_{\text {init }}$ ) taking into account the confirmed/suspected ratio obtained in each stream Total 4 gives the inferred fraction of stars in stream in the full sample of 2910 stars (Col. $N_{\text {init }}$ ). Total 5 is the fraction of stars in stream corrected for field contamination (Col. $N_{\text {init }}$ )

\begin{tabular}{|c|c|c|c|c|c|c|c|}
\hline Stream & $N_{\text {init }}$ & $N_{V_{\mathrm{R}}}$ & $\begin{array}{r}N_{\text {sel }} \\
(|\kappa|=3 .) \\
\end{array}$ & $\%_{\text {field }}$ & $\begin{array}{c}\bar{U} \pm \sigma_{u} \\
\left(\mathrm{~km} \mathrm{~s}^{-1}\right)\end{array}$ & $\begin{array}{l}\bar{V} \pm \sigma_{v} \\
\left(\mathrm{~km} \mathrm{~s}^{-1}\right)\end{array}$ & $\begin{array}{l}\bar{W} \pm \sigma_{w} \\
\left(\mathrm{~km} \mathrm{~s}^{-1}\right)\end{array}$ \\
\hline 3-8 Pleiades SCl & 554 & 276 & 254 & $23 \%$ & $-14.4 \pm 8.4$ & $-20.1 \pm 5.9$ & $-6.2 \pm 6.3$ \\
\hline $3-9$ & 23 & 7 & 4 & & $21.0 \pm 5.7$ & $-12.6 \pm 3.2$ & $-3.3 \pm 9.1$ \\
\hline 3-10 Hyades SCl & 287 & 140 & 126 & $34 \%$ & $-32.9 \pm 6.6$ & $-14.5 \pm 6.8$ & $-5.6 \pm 6.5$ \\
\hline $3-11$ & 10 & 7 & 5 & & $-34.8 \pm 3.1$ & $-14.4 \pm 2.5$ & $-17.6 \pm 1.7$ \\
\hline $3-14$ & 19 & 6 & 3 & & $32.8 \pm 3.0$ & $-6.0 \pm 2.0$ & $-9.6 \pm 1.1$ \\
\hline 3-15 Cen Associations & 577 & 254 & 228 & $20 \%$ & $-15.2 \pm 8.6$ & $-8.4 \pm 6.7$ & $-8.8 \pm 6.1$ \\
\hline $3-16$ & 21 & 8 & 3 & & $-2.0 \pm 1.6$ & $-7.1 \pm 2.4$ & $-2.5 \pm 4.8$ \\
\hline 3-18 New SCl & 406 & 197 & 158 & $23 \%$ & $3.6 \pm 6.8$ & $2.9 \pm 5.0$ & $-6.0 \pm 6.3$ \\
\hline 3-19 Sirius SCl & 195 & 97 & 92 & $31 \%$ & $14.0 \pm 7.3$ & $1.0 \pm 6.4$ & $-7.8 \pm 5.5$ \\
\hline Total 1 & 2092 & 992 & & & & & \\
\hline Total 2 & & & 873 & \multirow{2}{*}{\multicolumn{4}{|c|}{$\begin{array}{l}\left.\text { ( } V_{R} \text { confirmed stream members }\right) \\
\text { (Total stream members) }\end{array}$}} \\
\hline Total 3 & 1834.3 & & & & & & \\
\hline Total 4 & $\mathbf{6 3 . 0} \%$ & & & \multicolumn{4}{|c|}{ (Fraction of stars in streams in the full sample) } \\
\hline Total 5 & $\mathbf{4 6 . 4} \%$ & & & \multicolumn{4}{|c|}{ (Fraction of stars in streams corrected for field contamination) } \\
\hline
\end{tabular}

\section{Pleiades SCl}

The analysis of the Pleiades $\mathrm{SCl}$ is realized in Paper II where it is found to be composed of two main streams of few $10^{7}$ and $10^{9} \mathrm{yr}$.

\section{Hyades SCl and NGC 1901 stream}

The velocity clump (stream 3-10 in Table 2) identified at scale 3 as the Hyades SCl (see Figs. 15 for velocity and age distributions and Figs. 16 for space distributions) is located at $(U, V, W)=(-32.9$, $-14.5,-5.6) \mathrm{km} \mathrm{s}^{-1}$ with velocity dispersions $\left(\sigma_{U}\right.$, $\left.\sigma_{V}, \sigma_{W}\right)=(6.6,6.8,6.5) \mathrm{km} \mathrm{s}^{-1}$. The mean velocity deviates slightly from the definition given by Eggen (1992b) (cf. Table 5). At this resolution the bulk of star ages is between $410^{8} \mathrm{yr}$ and $210^{9} \mathrm{yr}$ with two peaks at $610^{8}$ and $1.610^{9} \mathrm{yr}$ in Strömgren age distribution plus a $10^{7}$ yr peak in the palliative age distribution. Eggen (1992b) pointed out that the supercluster contains at least three age groups around 3 to 4,6 and $810^{8} \mathrm{yr}$. The velocity pattern splits into 3 groups at scale 2 , namely 2-10, 2-18 and 2-25 (Table 3). Each stream presents a characteristic age distribution, although the velocity separation (centers deviates from each other by several $\mathrm{km} \mathrm{s}^{-1}$ in $\mathrm{W}$ ) does not produce a neat age separation. Three different main components of $10^{7}$, $5-610^{8}$ and $10^{9} \mathrm{yr}$ are mixed in the 3 clumps. The first clump peaks at $10^{9} \mathrm{yr}$ in Strömgren ages but contains a $610^{8}$ yr old component also revealed by palliative ages. The second clump peaks at $510^{8} \mathrm{yr}$ and $10^{9} \mathrm{yr}$ in Strömgren ages. Palliative ages produce a $10^{7}$ yr peak which is probably a statistical ghost of the $510^{8}$ year old component (see explanation of ghost at the end of Sect. 3.2). The third clump is dominated by a $510^{8}$ yr old component with two older groups of $10^{9}$ and $210^{9} \mathrm{yr}$. One more time the very young peak in palliative ages is also probably due to the $510^{8}$ year old component.

The presence of older supercluster members around $1.610^{9} \mathrm{yr}$ as stipulated by Eggen and stressed by Chen et al. (1997) is detected in the third velocity clump. Scale 1 does not reveal more information so that we cannot obtain one age for each stream.

So, the Hyades SCl contains probably three groups of $5-610^{8} \mathrm{yr}, 10^{9}$ and $1.6-210^{9} \mathrm{yr}$ which are in an advanced stage of dispersion in the same velocity volume. Only part of these 3 streams can be linked to the 
Table 3. Main characteristics of detected velocity structures at scale 2 with filter size of $5.5 \mathrm{~km} \mathrm{~s}^{-1}\left(\bar{\sigma}_{\text {stream }} \sim 3.8 \mathrm{~km} \mathrm{~s}{ }^{-1}\right)$. Legend is the same as Table 2

\begin{tabular}{|c|c|c|c|c|c|c|}
\hline Stream & $N_{\text {init }}$ & $N_{V_{\mathrm{R}}}$ & $\begin{array}{r}N_{\text {sel }} \\
(|\kappa|=3 .) \\
\end{array}$ & $\begin{array}{c}\bar{U} \pm \sigma_{u} \\
\left(\mathrm{~km} \mathrm{~s}^{-1}\right)\end{array}$ & $\begin{array}{c}\bar{V} \pm \sigma_{v} \\
\left(\mathrm{~km} \mathrm{~s}^{-1}\right)\end{array}$ & $\begin{array}{l}\bar{W} \pm \sigma_{w} \\
\left(\mathrm{~km} \mathrm{~s}^{-1}\right)\end{array}$ \\
\hline 2-2 Pleiades $\mathrm{OCl}$ & 89 & 50 & 37 & $-7.5 \pm 4.6$ & $-24.6 \pm 3.5$ & $-10.2 \pm 3.5$ \\
\hline 2-5 Pleiades SCl & 161 & 85 & 67 & $-12.0 \pm 5.3$ & $-21.6 \pm 4.7$ & $-5.3 \pm 5.9$ \\
\hline $2-7$ & 52 & 22 & 13 & $-27.3 \pm 5.6$ & $-17.8 \pm 1.4$ & $-8.5 \pm 5.4$ \\
\hline 2-10 Hyades SCl (1) & 67 & 33 & 23 & $-31.6 \pm 2.8$ & $-15.8 \pm 2.8$ & $0.8 \pm 2.7$ \\
\hline 2-12 Cen-Lup & 112 & 45 & 28 & $-12.4 \pm 6.1$ & $-16.5 \pm 4.6$ & $-7.4 \pm 3.1$ \\
\hline $2-13$ & 27 & 15 & 5 & $-32.3 \pm 4.9$ & $-16.3 \pm 2.2$ & $-15.3 \pm 4.6$ \\
\hline 2-14 IC $2391 \mathrm{SCl}$ & 149 & 66 & 50 & $-20.8 \pm 4.3$ & $-14.5 \pm 4.9$ & $-4.9 \pm 5.0$ \\
\hline 2-15 Hyades $\mathrm{OCl}$ & 26 & 14 & 8 & $-42.8 \pm 3.6$ & $-17.9 \pm 3.2$ & $-2.2 \pm 5.2$ \\
\hline 2-18 Hyades SCl (2) & 59 & 27 & 17 & $-33.0 \pm 4.2$ & $-14.1 \pm 4.0$ & $-5.1 \pm 3.1$ \\
\hline $2-23$ & 26 & 15 & 9 & $-2.9 \pm 1.9$ & $-10.6 \pm 2.4$ & $-5.3 \pm 5.0$ \\
\hline 2-25 Hyades SCl (3) & 49 & 29 & 17 & $-32.8 \pm 2.8$ & $-11.8 \pm 2.8$ & $-8.9 \pm 2.9$ \\
\hline 2-26 Cen-Crux & 273 & 116 & 93 & $-13.1 \pm 6.2$ & $-7.9 \pm 6.1$ & $-9.3 \pm 5.5$ \\
\hline $2-27$ New $\mathrm{SCl}(1)$ & 45 & 20 & 6 & $1.0 \pm 6.0$ & $-4.0 \pm 6.1$ & $0.2 \pm 4.2$ \\
\hline 2-29 NGC 1901 & 33 & 14 & 5 & $-26.1 \pm 2.1$ & $-7.6 \pm 3.7$ & $0.7 \pm 3.2$ \\
\hline $2-31$ & 28 & 16 & 7 & $-5.8 \pm 4.5$ & $-0.8 \pm 6.6$ & $-15.6 \pm 4.1$ \\
\hline $2-32$ & 20 & 12 & 6 & $19.3 \pm 1.9$ & $-5.1 \pm 5.3$ & $-11.9 \pm 2.6$ \\
\hline $2-33$ & 16 & 8 & 4 & $20.1 \pm 1.6$ & $-2.6 \pm 1.4$ & $-15.9 \pm 4.8$ \\
\hline $2-34$ & 15 & 7 & 6 & $21.1 \pm 2.4$ & $0.4 \pm 3.9$ & $-0.8 \pm 2.9$ \\
\hline 2-35 New SCl (2) & 89 & 40 & 28 & $4.5 \pm 5.4$ & $-0.9 \pm 4.4$ & $-7.4 \pm 2.7$ \\
\hline 2-37 Sirius SCl (1) & 64 & 34 & 27 & $12.4 \pm 4.0$ & $0.7 \pm 4.6$ & $-7.7 \pm 4.7$ \\
\hline 2-38 New SCl (3) & 102 & 51 & 23 & $1.3 \pm 4.8$ & $3.0 \pm 2.3$ & $-3.6 \pm 3.1$ \\
\hline $2-40$ & 16 & 8 & 3 & $-4.8 \pm 3.1$ & $5.2 \pm 6.7$ & $-11.3 \pm 3.6$ \\
\hline 2-41 Sirius SCl (2) & 42 & 21 & 13 & $12.4 \pm 3.7$ & $4.2 \pm 3.3$ & $-9.0 \pm 2.9$ \\
\hline 2-43 New SCl (4) & 77 & 46 & 28 & $5.3 \pm 2.7$ & $6.5 \pm 3.6$ & $-11.4 \pm 4.0$ \\
\hline 2-44 New SCl (5) & 45 & 20 & 8 & $6.3 \pm 2.8$ & $6.5 \pm 2.6$ & $0.8 \pm 2.7$ \\
\hline $2-46$ & 24 & 13 & 7 & $-4.8 \pm 6.1$ & $10.8 \pm 2.0$ & $-6.5 \pm 1.9$ \\
\hline Total 1 & 1706 & 827 & & & & \\
\hline Total 2 & 143 & & 538 & \multicolumn{3}{|c|}{$\begin{array}{l}\text { ( } V_{R} \text { confirmed stream members) } \\
\text { (Total stream members) }\end{array}$} \\
\hline Total 4 & $\mathbf{3 8 . 3} \%$ & & & \multicolumn{3}{|c|}{ (Fraction of stars in streams) } \\
\hline
\end{tabular}

evaporation of known open clusters. The Hyades $\mathrm{OCl}$ recent evaporation is clearly found separately in stream $2-15$. The Praesepe OCl mean velocity (Table 5) accurately match none of the 3 stream velocities but could explain the stream 2-18 despite a difference of $\sim 9 \mathrm{~km} \mathrm{~s}^{-1}$ in the $V$ component. The NGC $1901 \mathrm{su}$ percluster described in Eggen, 1996 and assumed to be a Hyades $\mathrm{SCl}$ component is found separately at scale 2 (stream 2-29 in Table 3) and exhibits a single mode in age distribution at $810^{8} \mathrm{yr}$. Its velocity is more dissociated from the supercluster mean velocity than the 3 other streams which explain a best member extraction.

\section{Sirius SCl}

The Sirius supercluster (see Figs. 17 for velocity and age distributions and Figs. 18, 19 for space distributions) is found on scale 3 (stream 3-19 in Table 4 ) at mean velocity $(U, V, W)=(+14.0,+1.0,-7.8)$ $\mathrm{km} \mathrm{s}^{-1}$ with velocity dispersions $\left(\sigma_{U}, \sigma_{V}, \sigma_{W}\right)=$
$(7.3,6.4,5.5) \mathrm{km} \mathrm{s}^{-1}$. Eggen (1992c) identifies two age groups, $6.310^{8}$ and $10^{9} \mathrm{yr}$ and notices that there are also younger $\left(\begin{array}{lll}2.5 & 10^{8} & \mathrm{yr}\end{array}\right)$ and older members $\left(1.510^{9} \mathrm{yr}\right)$. At the coarse resolution (scale 3$)$, the age distribution is in relative good agreement with this description: Strömgren ages peak at $610^{8}$ yr and there is a significant proportion of stars between $10^{9}$ and $210^{9}$ yr. Stars younger than $2.510^{8} \mathrm{yr}$ are probably not a statistical ghost of the $610^{8}$ year old component since some stars with Strömgren ages are also present. At scale 2, the supercluster splits into two distinct streams (stream 2-37 and 2-41 in Table 3) at respectively $(U, V, W)=(+12.4,+0.7,-7.7) \mathrm{km} \mathrm{s}^{-1}$ with $\left(\sigma_{U}, \sigma_{V}, \sigma_{W}\right)=(4.0,4.6,4.7) \mathrm{km} \mathrm{s}^{-1}$ and $(U, V, W)=$ $(+12.4,+4.2,-9.0) \mathrm{km} \mathrm{s}^{-1}$ with $\left(\sigma_{U}, \sigma_{V}, \sigma_{W}\right)=(3.7$, $3.3,2.9) \mathrm{km} \mathrm{s}^{-1}$ producing a very clear age separation: the very young stars are separated from a part of the oldest components ( $610^{8}$ and $1.610^{9} \mathrm{yr}$ ). The very young component appears exclusively in stream 2-37 
Table 4. Main characteristics of detected velocity structures at scale 1 with typical size of $3.2 \mathrm{~km} \mathrm{~s}^{-1}\left(\bar{\sigma}_{\text {stream }} \sim 2.4 \mathrm{~km} \mathrm{~s}^{-1}\right)$. Legend is the same as Table 2

\begin{tabular}{|c|c|c|c|c|c|c|}
\hline Stream & $N_{\text {init }}$ & $N_{V_{\mathrm{R}}}$ & $\begin{array}{r}N_{\text {sel }} \\
(|\kappa|=3 .) \\
\end{array}$ & $\begin{array}{l}\bar{U} \pm \sigma_{u} \\
\left(\mathrm{~km} \mathrm{~s}^{-1}\right)\end{array}$ & $\begin{array}{l}\bar{V} \pm \sigma_{v} \\
\left(\mathrm{~km} \mathrm{~s}^{-1}\right)\end{array}$ & $\begin{array}{l}\bar{W} \pm \sigma_{w} \\
\left(\mathrm{~km} \mathrm{~s}^{-1}\right)\end{array}$ \\
\hline $1-1$ & 14 & 7 & 3 & $-14.9 \pm 0.5$ & $-27.8 \pm 0.6$ & $-1.7 \pm 1.9$ \\
\hline 1-2 Pleiades $\mathrm{OCl}$ & 40 & 17 & 12 & $-4.7 \pm 2.0$ & $-23.8 \pm 1.2$ & $-8.9 \pm 2.6$ \\
\hline $1-4$ & 16 & 6 & 3 & $-15.1 \pm 3.1$ & $-24.1 \pm 2.9$ & $-0.7 \pm 1.7$ \\
\hline 1-6 Pleiades SCl (1) & 33 & 14 & 9 & $-13.1 \pm 3.1$ & $-21.9 \pm 3.3$ & $-7.1 \pm 2.5$ \\
\hline 1-7 Pleiades SCl (2) & 46 & 30 & 20 & $-11.1 \pm 1.7$ & $-21.9 \pm 3.0$ & $-6.0 \pm 1.8$ \\
\hline $1-10$ & 22 & 10 & 3 & $-31.0 \pm 5.2$ & $-19.0 \pm 1.5$ & $-6.0 \pm 2.7$ \\
\hline $1-11$ & 18 & 6 & 3 & $-19.2 \pm 3.4$ & $-19.0 \pm 2.4$ & $-7.1 \pm 3.5$ \\
\hline $1-12$ & 22 & 11 & 3 & $-9.9 \pm 0.4$ & $-20.3 \pm 1.6$ & $-0.5 \pm 1.8$ \\
\hline $1-13$ & 22 & 11 & 3 & $-16.1 \pm 2.0$ & $-20.9 \pm 0.1$ & $1.9 \pm 2.9$ \\
\hline $1-15$ & 32 & 17 & 9 & $-18.8 \pm 4.2$ & $-15.7 \pm 3.4$ & $-1.8 \pm 2.3$ \\
\hline 1-17 Cen-Lup & 61 & 17 & 5 & $-9.4 \pm 3.3$ & $-18.2 \pm 2.7$ & $-5.9 \pm 1.1$ \\
\hline 1-19 Hyades SCl (1) & 24 & 12 & 10 & $-29.0 \pm 5.5$ & $-12.5 \pm 6.0$ & $-0.0 \pm 4.4$ \\
\hline $1-20$ IC $2391 \mathrm{SCl}(1)$ & 33 & 15 & 8 & $-20.1 \pm 2.9$ & $-12.8 \pm 3.1$ & $-5.0 \pm 1.8$ \\
\hline $1-21$ & 8 & 6 & 3 & $-19.9 \pm 1.1$ & $-14.8 \pm 3.2$ & $-15.5 \pm 0.5$ \\
\hline $1-22$ & 28 & 12 & 3 & $-27.2 \pm 2.4$ & $-17.1 \pm 2.8$ & $-8.4 \pm 3.2$ \\
\hline 1-23 Hyades SCl (2) & 39 & 22 & 8 & $-33.2 \pm 2.3$ & $-13.1 \pm 3.5$ & $-6.6 \pm 2.5$ \\
\hline $1-24$ & 32 & 12 & 3 & $-13.8 \pm 2.7$ & $-15.5 \pm 5.9$ & $1.1 \pm 3.4$ \\
\hline $1-25$ IC $2391 \mathrm{SCl}(2)$ & 41 & 21 & 8 & $-21.0 \pm 4.5$ & $-10.0 \pm 3.7$ & $-6.1 \pm 2.9$ \\
\hline $1-26$ & 23 & 11 & 6 & $-2.4 \pm 1.6$ & $-11.7 \pm 1.8$ & $-4.5 \pm 3.2$ \\
\hline $1-27$ & 12 & 7 & 3 & $-30.5 \pm 1.0$ & $-10.8 \pm 0.6$ & $-9.1 \pm 2.1$ \\
\hline 1-29 Cen-Crux & 45 & 17 & 6 & $-13.1 \pm 3.2$ & $-9.4 \pm 3.7$ & $-7.7 \pm 4.5$ \\
\hline $1-31$ & 53 & 21 & 12 & $-19.5 \pm 3.4$ & $-5.8 \pm 2.3$ & $-10.5 \pm 3.0$ \\
\hline $1-32$ & 17 & 7 & 4 & $-25.8 \pm 2.0$ & $-9.9 \pm 3.2$ & $-0.2 \pm 3.0$ \\
\hline $1-34$ & 24 & 11 & 7 & $-8.0 \pm 1.0$ & $-3.8 \pm 1.3$ & $-13.2 \pm 2.2$ \\
\hline $1-37$ & 14 & 9 & 3 & $3.1 \pm 5.4$ & $-1.1 \pm 1.8$ & $-15.2 \pm 3.4$ \\
\hline $1-39$ & 13 & 6 & 5 & $22.1 \pm 1.5$ & $-1.1 \pm 1.9$ & $-0.5 \pm 2.2$ \\
\hline 1-41 New SCl (2) & 39 & 18 & 11 & $4.1 \pm 4.2$ & $0.0 \pm 2.4$ & $-7.7 \pm 1.8$ \\
\hline $1-45$ & 14 & 5 & 3 & $8.5 \pm 1.4$ & $0.9 \pm 1.3$ & $-2.4 \pm 2.5$ \\
\hline $1-46$ & 27 & 13 & 4 & $1.4 \pm 4.3$ & $1.4 \pm 2.0$ & $-3.4 \pm 2.0$ \\
\hline $1-49$ & 16 & 6 & 3 & $15.6 \pm 1.3$ & $2.1 \pm 4.4$ & $-9.1 \pm 1.6$ \\
\hline 1-51 Sirius SCl (2) & 16 & 7 & 4 & $8.9 \pm 3.1$ & $4.8 \pm 1.6$ & $-12.9 \pm 0.6$ \\
\hline $1-54$ & 18 & 11 & 5 & $6.5 \pm 1.2$ & $5.9 \pm 1.8$ & $-10.6 \pm 2.1$ \\
\hline 1-55 New SCl (3) & 18 & 9 & 5 & $2.9 \pm 4.4$ & $3.9 \pm 1.8$ & $-4.9 \pm 2.6$ \\
\hline 1-56 Sirius SCl (1) & 13 & 8 & 4 & $12.0 \pm 2.7$ & $5.9 \pm 1.3$ & $-9.2 \pm 1.1$ \\
\hline $1-58$ & 20 & 11 & 6 & $3.7 \pm 1.9$ & $7.2 \pm 3.5$ & $-10.0 \pm 2.7$ \\
\hline 1-59 New SCl (5) & 24 & 9 & 4 & $6.2 \pm 0.6$ & $6.0 \pm 1.2$ & $-0.3 \pm 1.9$ \\
\hline $1-61$ New $\mathrm{SCl}(4)$ & 15 & 5 & 4 & $7.2 \pm 1.8$ & $9.9 \pm 2.2$ & $-10.5 \pm 2.7$ \\
\hline $1-62$ & 17 & 9 & 5 & $-3.9 \pm 7.6$ & $10.5 \pm 2.2$ & $-7.2 \pm 1.1$ \\
\hline 1-59 New SCl (5) & 24 & 9 & 4 & $6.2 \pm 0.6$ & $6.0 \pm 1.2$ & $-0.3 \pm 1.9$ \\
\hline $1-61$ New $\mathrm{SCl}(4)$ & 15 & 5 & 4 & $7.2 \pm 1.8$ & $9.9 \pm 2.2$ & $-10.5 \pm 2.7$ \\
\hline $1-62$ & 17 & 9 & 5 & $-3.9 \pm 7.6$ & $10.5 \pm 2.2$ & $-7.2 \pm 1.1$ \\
\hline Total 1 & 1262 & 556 & & & & \\
\hline Total 2 & & & 220 & \multicolumn{3}{|c|}{ ( $V_{R}$ confirmed stream members) } \\
\hline Total 3 & 521.2 & & & \multicolumn{3}{|c|}{ (Total stream members) } \\
\hline Total 4 & $17.9 \%$ & & & \multicolumn{3}{|c|}{ (Fraction of stars in streams) } \\
\hline
\end{tabular}


E. Chereul et al.: The distribution of nearby stars in phase space mapped by Hipparcos. III.

Table 5. Cross-identification data for known kinematical groups: Open clusters $(\mathrm{OCl})$ and Superclusters $(\mathrm{SCl})$

\begin{tabular}{|c|c|c|c|c|c|}
\hline & $\bar{U}$ & $\begin{array}{c}\bar{V} \\
\left(\mathrm{~km} \mathrm{~s}^{-1}\right)\end{array}$ & $\bar{W}$ & $\begin{array}{l}\text { Age } \\
(\mathrm{yr})\end{array}$ & Source \\
\hline \multicolumn{6}{|l|}{$\mathrm{OCl}$} \\
\hline $\begin{array}{l}\text { Hyades } \\
\text { Pleiades } \\
\text { IC2391 } \\
\text { U.Ma } \\
\text { Coma Ber. } \\
\text { IC2602 } \\
\text { Praesepe } \\
\text { Alpha Per. }\end{array}$ & $\begin{array}{r}-44.4 \\
-5.8 \\
-18.3 \\
+14.5 \\
-1.8 \\
-0.7 \\
-37.1 \\
-10.8 \\
\end{array}$ & $\begin{array}{r}-17.0 \\
-24.0 \\
-13.5 \\
+2.5 \\
-8.2 \\
-25.7 \\
-23.5 \\
-20.5 \\
\end{array}$ & $\begin{array}{r}-5.0 \\
-12.4 \\
-5.9 \\
-8.5 \\
-0.7 \\
-1.4 \\
-7.0 \\
-0.7 \\
\end{array}$ & $\begin{array}{c}8 \pm 210^{8} \\
5 \pm 110^{7} \\
3 \pm 110^{7} \\
310^{8} \\
4 \pm 110^{8} \\
1 \pm 0.510^{7} \\
7 \pm 210^{8} \\
4 \pm 0.510^{7} \\
\end{array}$ & $\begin{array}{l}\text { Pallous̀, et al., } 1977 \\
\text { Pallous̀, et al., } 1977 \\
\text { Pallous̀, et al., } 1977 \\
\text { Eggen, } 1973 \\
\text { Pallous̀, et al., } 1977 \\
\text { Pallous̀, et al., } 1977 \\
\text { Pallous̀, et al., } 1977 \\
\text { Pallous̀, et al., } 1977\end{array}$ \\
\hline \multicolumn{6}{|l|}{$\mathrm{SCl}$} \\
\hline $\begin{array}{l}\text { Hyades } \\
\text { Hyades }\end{array}$ & $\begin{array}{l}-40.0 \\
-44.4\end{array}$ & $\begin{array}{l}-17.0 \\
-17.8\end{array}$ & $\begin{array}{l}-2.0 \\
-1.5\end{array}$ & $\begin{array}{c}3-6-810^{8} \\
13.310^{8}\end{array}$ & $\begin{array}{l}\text { Eggen, 1992b } \\
\text { Chen, et al., } 1997\end{array}$ \\
\hline $\begin{array}{l}\text { Pleiades } \\
\text { Pleiades }\end{array}$ & $\begin{array}{l}-11.6 \\
-10.0\end{array}$ & $\begin{array}{l}-20.7 \\
-19.0\end{array}$ & $\begin{array}{r}-10.4 \\
-8.1\end{array}$ & $\begin{array}{c}510^{7} \& 210^{8} \\
0.710^{8}\end{array}$ & $\begin{array}{l}\text { Eggen, 1992a } \\
\text { Chen, et al., } 1997\end{array}$ \\
\hline $\begin{array}{l}\text { IC } 2391 \\
\text { IC } 2391 \\
\text { IC } 2391\end{array}$ & $\begin{array}{l}-22.4 \\
-20.8 \\
-15.9\end{array}$ & $\begin{array}{l}-17.5 \\
-15.9 \\
-13.1\end{array}$ & $\begin{array}{l}-9.4 \\
-8.3 \\
-4.5\end{array}$ & $\begin{array}{c}810^{7} \& 2.510^{8} \\
2.810^{8}\end{array}$ & $\begin{array}{l}\text { Eggen, 1991b } \\
\text { Eggen, 1992d } \\
\text { Chen, et al., } 1997\end{array}$ \\
\hline $\begin{array}{l}\text { Sirius } \\
\text { Sirius }\end{array}$ & $\begin{array}{l}+15.0 \\
+13.0\end{array}$ & $\begin{array}{l}+1.0 \\
+3.2\end{array}$ & $\begin{array}{r}-11.0 \\
-7.5\end{array}$ & $\begin{array}{c}6.310^{8} \& 10^{9} \\
6.510^{8}\end{array}$ & $\begin{array}{l}\text { Eggen, 1992c } \\
\text { Chen, et al., } 1997\end{array}$ \\
\hline NGC 1901 & -26.4 & -10.4 & -1.5 & $810^{8} \& 1.210^{9}$ & Eggen, 1996 \\
\hline
\end{tabular}

(middle right of Fig. 17). At the highest resolution, on scale 1 (bottom of Fig. 17), the stream 2-41 contains oldest components still interpenetrated. Space distributions (Fig. 19) show that the first stream, which contains the $10^{7}$ year old component is still concentrated. There are too few members in the second clump to make conclusions.

The Sirius $\mathrm{SCl}$ is composed by three age components of $\sim 10^{7}, 610^{8}$ and $1.510^{9}$. The younger stream is still concentrated both kinematically and spatially while the two oldest streams are mixed in a larger volume of the phase space.

\section{IC $2391 \mathrm{SCl}$}

The IC 2391 SCl (see Figs. 20 for age distributions and Fig. 21 for space distributions) is not found at large scale because it may have been merged into the Centaurus association velocity group. It appears separately at scale 2 (stream $2-14$ in Table 3 ) at $(U$, $V, W)=(-20.8,-14.5,-4.9) \mathrm{km} \mathrm{s}^{-1}$ with velocity dispersions $\left(\sigma_{U}, \sigma_{V}, \sigma_{W}\right)=(4.3,4.9,5.0) \mathrm{km} \mathrm{s}^{-1}$ (see Fig. 15 of Paper II). Eggen (1991) states that

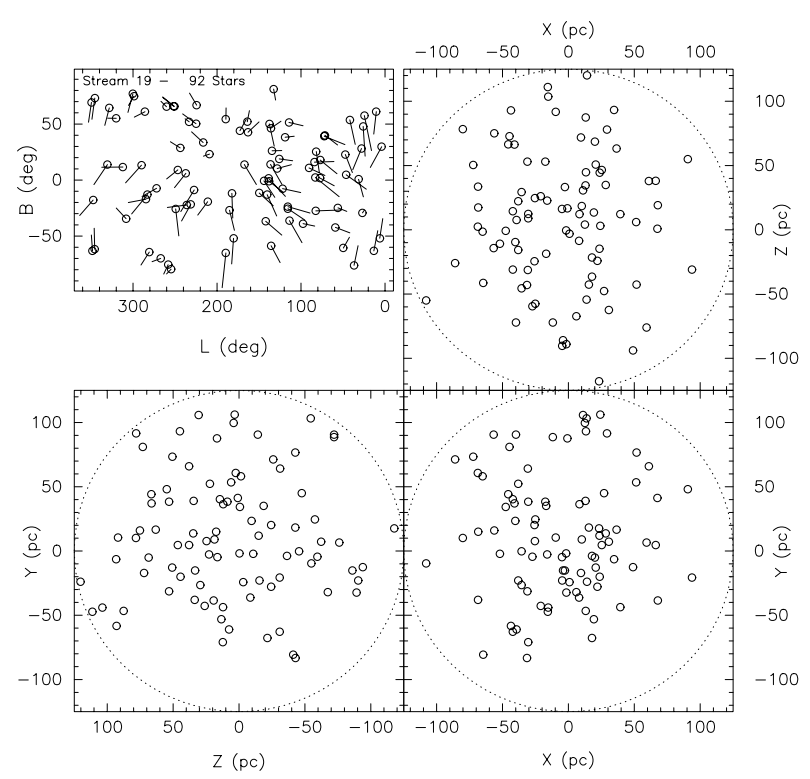

Fig. 18. Space distribution of Sirius $\mathbf{S C l}$ from the $V_{R}$ selected sub-sample at scale 3 (stream 3-19 in Table 2) 


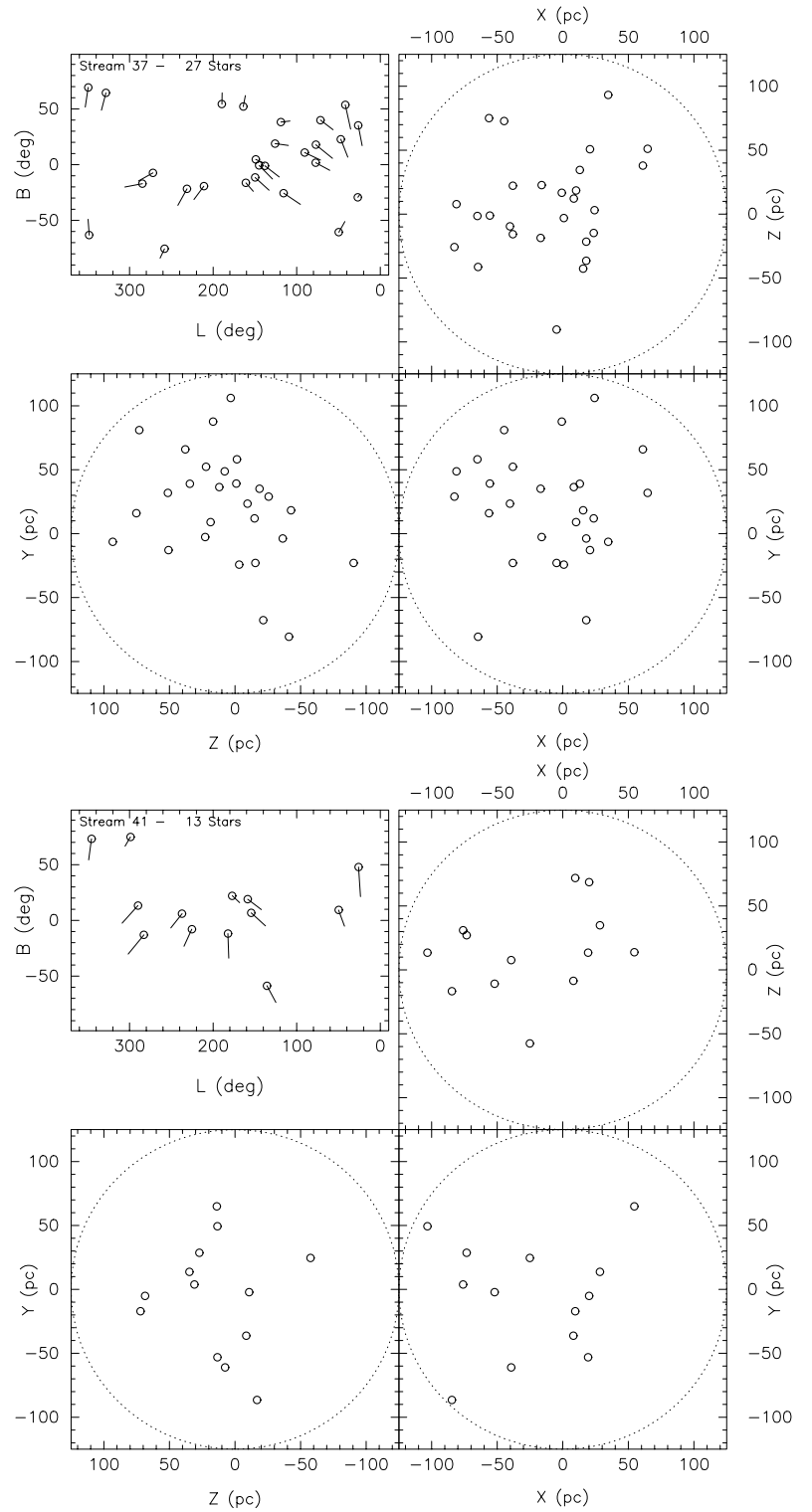

Fig. 19. Space distributions of the 2 sub-streams of Sirius SCl from the $V_{R}$ selected sub-sample at scale 2: stream 2-37 (top) and 2-41 in Table 3 (bottom)

IC $2391 \mathrm{SCl}$ contains two ages: $810^{7}$ and $2.510^{8} \mathrm{yr}$ while Chen et al. (1997) found a mean age of $4.6 \pm$ $1.610^{8} \mathrm{yr}$. The Strömgren age distribution is quite different from the palliative age distribution at coarser resolution. Strömgren ages peak at $810^{8}$ but with ages up to $210^{9} \mathrm{yr}$. Palliative ages exhibit a peak at $610^{8} \mathrm{yr}$ and a $10^{7}$ year old component (Fig. 20). This last peak is certainly real because its proportion is too high to be a statistical ghost of palliative ages from a $610^{8}$ year old component and moreover Strömgren ages show the presence of young stars. Two sub-streams are found at scale 1 (stream 1-20 and 1-25 in Table 4) at $(U$, $V, W)=(-20.1,-12.8,-5.0)$ with $\left(\sigma_{U}, \sigma_{V}, \sigma_{W}\right)=$ $(2.9,3.1,1.8) \mathrm{km} \mathrm{s}^{-1}$ and $(U, V, W)=(-20.9,-10.0$,
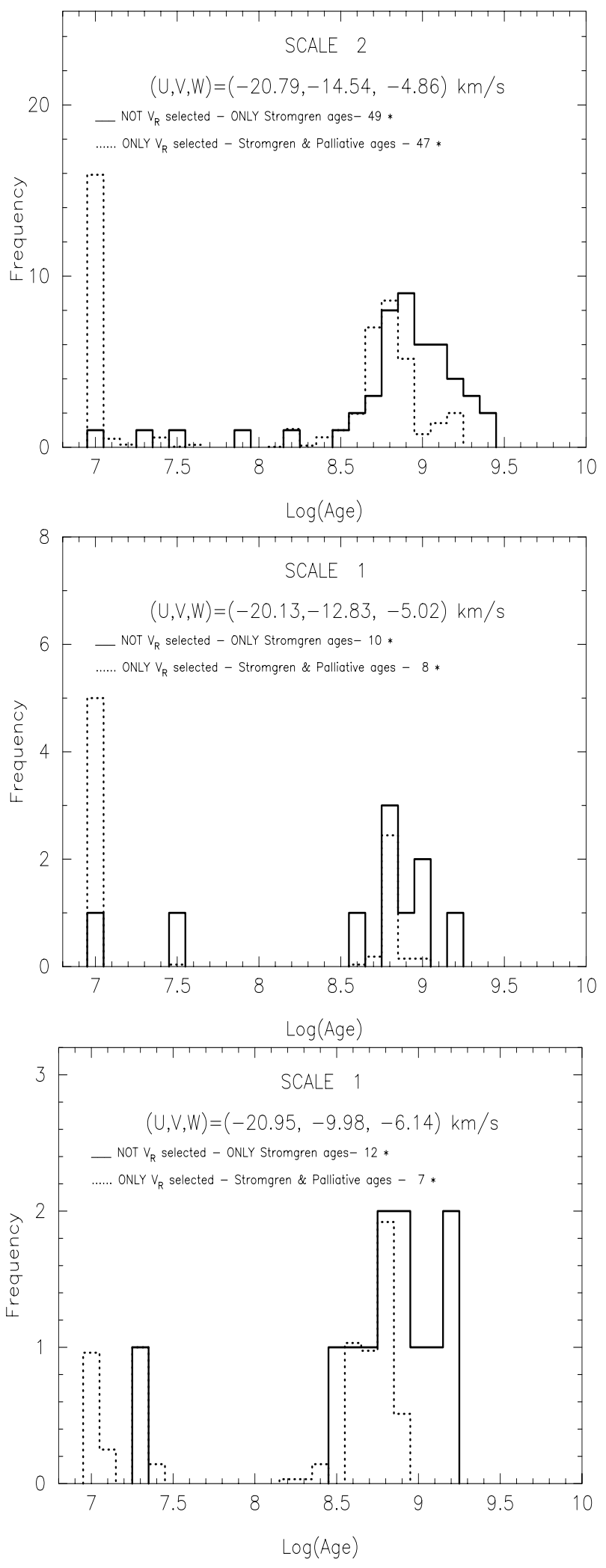

Fig. 20. IC 2391 SCl. Age distributions for the IC $2391 \mathrm{SCl}$ (stream 2-14 in Table 3) at scale 2 (top), for sub-stream 1-20 (in Table 4) at scale 1 (middle) and for sub-stream 1-25 (in Table 4) at scale 1 (bottom) 


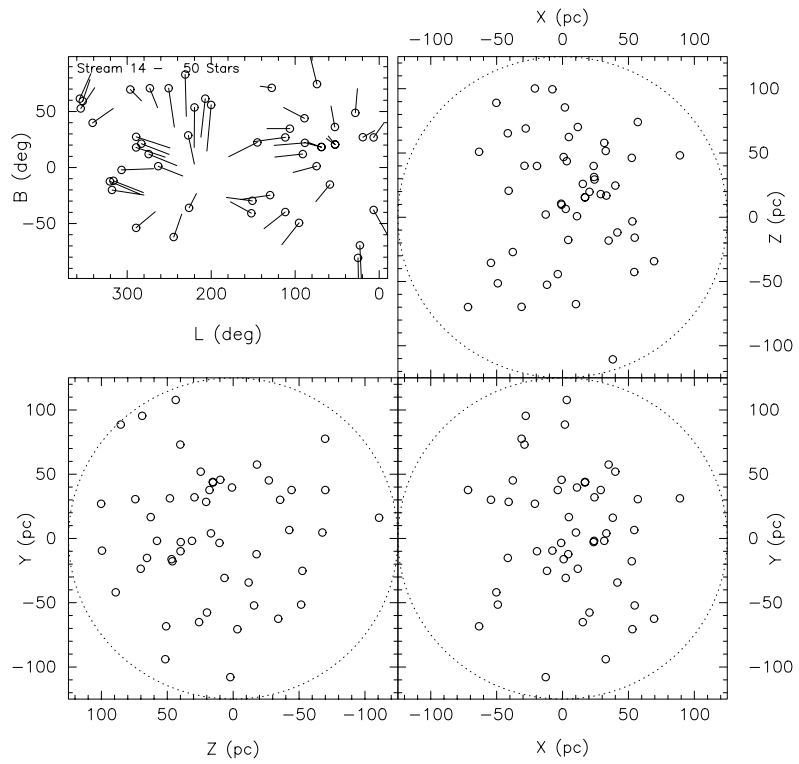

Fig. 21. Space distribution of IC $2391 \mathrm{SCl}$ from the $V_{R}$ selected sub-sample at scale 2 (stream 2-14 in Table 3 )

$-6.1)$ with $\left(\sigma_{U}, \sigma_{V}, \sigma_{W}\right)=(4.5,3.7,2.9) \mathrm{km} \mathrm{s}^{-1}$. The stream 1-20 contains all the youngest stars while the sub-stream 1-25 is only constituted of the $610^{8}$ year old population. Velocity dispersions of the two streams are in agreement with this view: they are smaller for the stream with the younger component. This configuration is exactly the opposite of the Pleiades' one: in this case the youngest population is more concentrated in the velocity space and is entirely detected in one stream while the oldest span over the two streams.

\section{Centaurus Associations and the Gould belt}

Lower Centaurus-Crux and upper Centaurus-Lupus associations (see Figs. 22 for age distributions and Figs. 23 for space distributions) which are the main components of the entire Centaurus association are detected as one velocity clump at scale 3 (stream 3-15 in Table 2) with $(U, V, W)=(-15.2,-8.4,-8.8) \mathrm{km} \mathrm{s}^{-1}$ with velocity dispersion $\left(\sigma_{U}, \sigma_{V}, \sigma_{W}\right)=(8.6,6.7,6.1)$ $\mathrm{km} \mathrm{s}^{-1}$. Scale 3 is a too coarse resolution and the age distributions reflect the overall distribution. The whole Centaurus association is splitted into two parts at scale 2 and does not evolve at scale 1 (see Paper II, Fig. 15).

At scale 2, Centaurus-Crux (stream 2-26 in Table 3) and Centaurus-Lupus (stream 2-12 in Table 3) are identified at $(U, V, W)=(-13.1,-7.9,-9.3) \mathrm{km} \mathrm{s}^{-1}$ with $\left(\sigma_{U}, \sigma_{V}, \sigma_{W}\right)=(6.2,6.1,5.5) \mathrm{km} \mathrm{s}^{-1}$ and $(U, V$, $W)=(-12.4,-16.5,-7.4) \mathrm{km} \mathrm{s}^{-1}$ with $\left(\sigma_{U}, \sigma_{V}, \sigma_{W}\right)$ $=(6.1,4.6,3.1) \mathrm{km} \mathrm{s}^{-1}$ respectively. Unfortunately, as for the Pleiades SCl, a lot of young stars have not Strömgren photometry. Strömgren age distributions peak at $610^{8} \mathrm{yr}$ for both sub-streams but palliative age distributions show the predominance of the very young population $\left(10^{7} \mathrm{yr}\right)$ in each case.
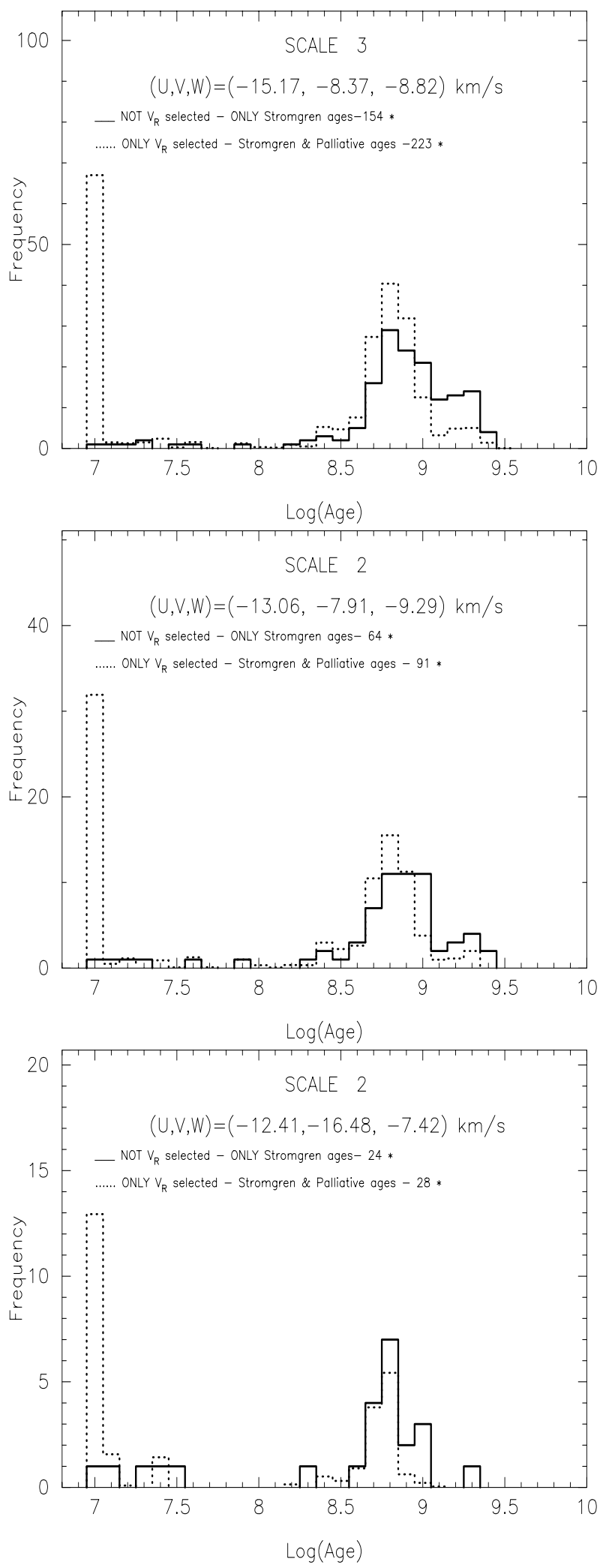

Fig. 22. Streams associated with Centaurus Associations. Age distributions of the overall association (stream 3-15 in Table 2) at scale 3 (top). Age distributions of Centaurus-Crux (stream $2-26$ in Table 3 ) at scale 2 (middle). Age distributions of Centaurus-Lupus (stream 2-12 in Table 3 ) at scale 2 (bottom) 
E. Chereul et al.: The distribution of nearby stars in phase space mapped by Hipparcos. III.
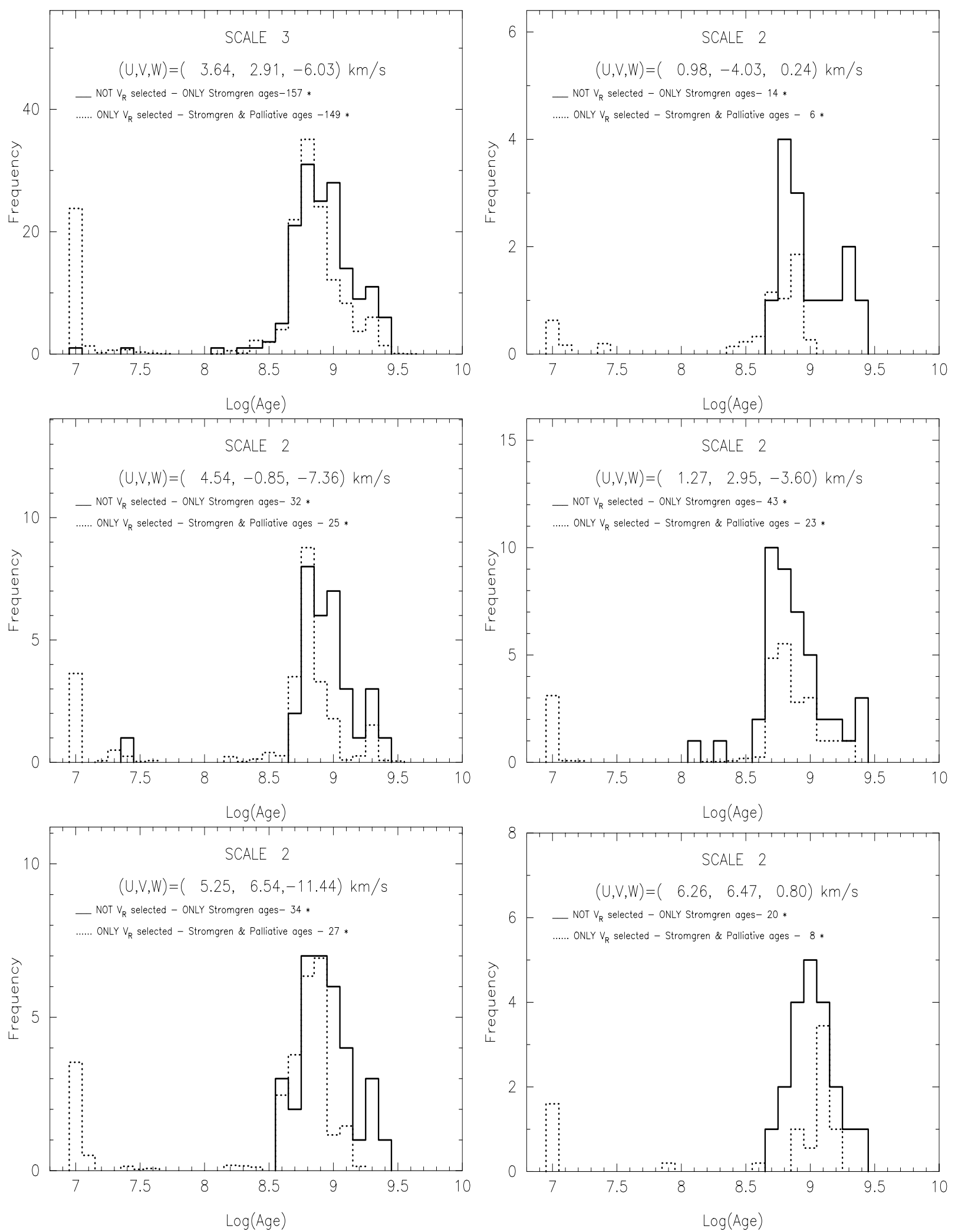

Fig. 25. New supercluster. Age distributions of the new moving group (stream 3-18 in Table 3) at scale 3 (top left) and the sub-stream 2-27 (in Table 3) at scale 2 (top right). Age distributions of the sub-streams 2-35 (middle left) and 2-38 (middle right) at scale 2. Age distributions of the sub-streams 2-43 (bottom left) and 2-44 (bottom right) at scale 2 

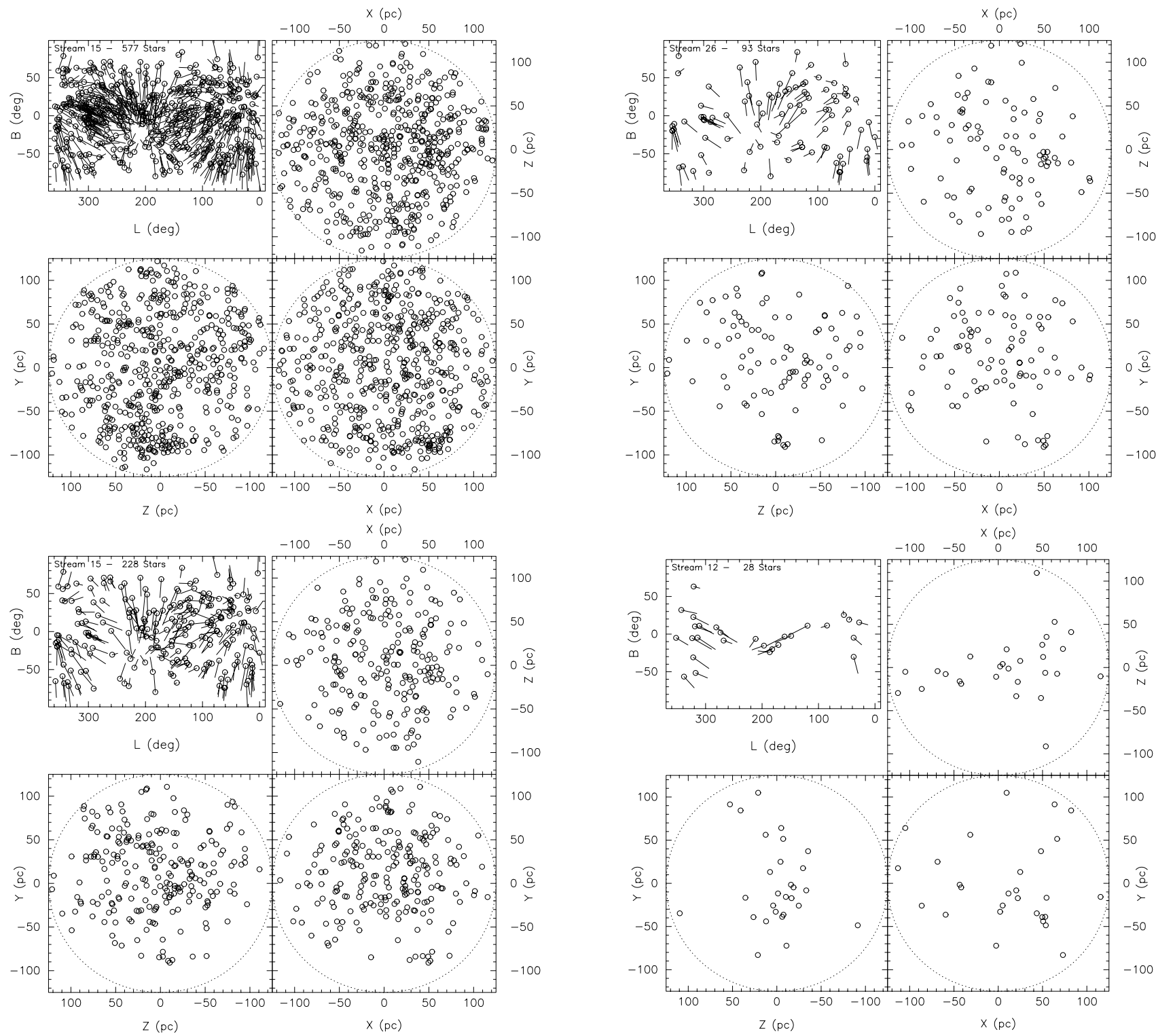

Fig. 23. Space distributions of the stream associated with Centaurus Associations (stream 3-15 in Table 2) for all the stars (top) and for the $V_{R}$ selected sub-sample (bottom) at scale 3. Stars belonging to spatial clumps in the upper figure disappear because of the lack of observed radial velocities

There is a crucial lack of radial velocities for the spatially clustered structures Centaurus-Crux and Centaurus-Lupus: one fifth of stars have observed $V_{R}$. That is why these space clumps are visible on space distributions when taking into account all the stars of the detected streams but disappear with the sub-sample selected on observed $V_{R}$ (Fig. 23). At scale 2, space distributions show that stars of the velocity substructure identified as Centaurus-Lupus association belong to a disk-like structure (see $X Z$ projection in Fig. 24) tilted with respect to the Galactic disk. Eigenvectors of the spatial ellipsoid

are calculated. The two vectors associated with the largest eigenvalues allow to define the plane of the structure, assuming it passes through the Sun. The ascending node of the intersection between this disk-like structure and the Galactic plane is $l_{\Omega}=317^{\circ}$ which differs slightly from usual values $\left(l_{\Omega} \sim 275^{\circ}\right.$ to $290^{\circ}$, Pöppel 1997). The angle between the two planes is $i=18.8^{\circ}$ in agreement with previous study $\left(i=18^{\circ}-23^{\circ}\right)$. 


\section{A new supercluster}

Close to the Sirius $\mathrm{SCl}$ in velocity space, located at the mean velocity $(U, V, W)=(+3.6,+2.9,-6.0)$ $\mathrm{km} \mathrm{s}^{-1}$ with velocity dispersions $\left(\sigma_{U}, \sigma_{V}, \sigma_{W}\right)=(6.8$, $5.0,6.3) \mathrm{km} \mathrm{s}^{-1}$, a new massive supercluster (stream 3-18 in Table 2) is detected at scale 3 (see Figs. 25 for age distributions and Fig. 26 for spatial distribution). It contains almost twice as many members as the Sirius SCl. None of the previously known superclusters corresponds to this velocity definition. Figueras et al. (1997) indicate the presence of a velocity structure at $(U, V)=(+7,+6)$ which they cannot confirm without doubt by their analysis and interpreted it as a possible sub-structure of Sirius SCl with a mean age of $10^{9} \mathrm{yr}$. We confirm the existence of a supercluster like structure, probably never detected before because of its low velocity with respect to the Sun. On a kinematics basis it is clearly dissociated from the Sirius $\mathrm{SCl}$.

Age distributions at coarser scale are similar to the whole sample ones with ages ranging from $10^{7}$ to $2.510^{9} \mathrm{yr}$. But at least 5 sub-streams at scale 2 (see Table 3) are found to form this structure. These streams show age distributions of relatively old components. Stream 2-27 shows an unambiguous peak at $610^{8}$ yr with few $1.610^{9}$ year old stars. On the basis of velocity and age content, this stream could originate from the evaporation of the Coma OCl. Stream 2-35 has stars which are $610^{8}, 10^{9}$ and $1.610^{9}$ year old on the basis of Strömgren photometry but palliative ages exhibit only one peak at $610^{8}$ yr. Stream 2-38 shows a peak at $510^{8}$ yr. Stream 2-43 has Strömgren ages between $6-810^{8}$ and few $1.610^{9}$ year old stars but the palliative ages exhibit only one peak at $810^{8}$. Stream $2-44$ is clearly a $10^{9}$ year old group. All the few very young palliative ages in each stream are probably statistical ghost because very young Strömgren ages are never present.

Age distributions at the highest resolution (scale 1), not shown here, give exactly the same results as scale 2 but the number of stars dramatically decreases.

This structure has the same features as the previously known superclusters: a juxtaposition of several little star formation bursts at different epochs in adjacent cells of the velocity field. The correlation between velocity and age is not always obvious because these bursts $\left(5-610^{8}, 810^{8}\right.$ and $\left.10^{9} \mathrm{yr}\right)$ are not very recent. As in the Hyades $\mathrm{SCl}$ case, stream velocity volumes, defined by their velocity dispersions, are substantially recovering.

Implications of these results on the understanding of the supercluster concept are discussed in Paper II.

\subsubsection{Outside superclusters}

- Small scale streams.

While superclusters are found to split into smaller scale

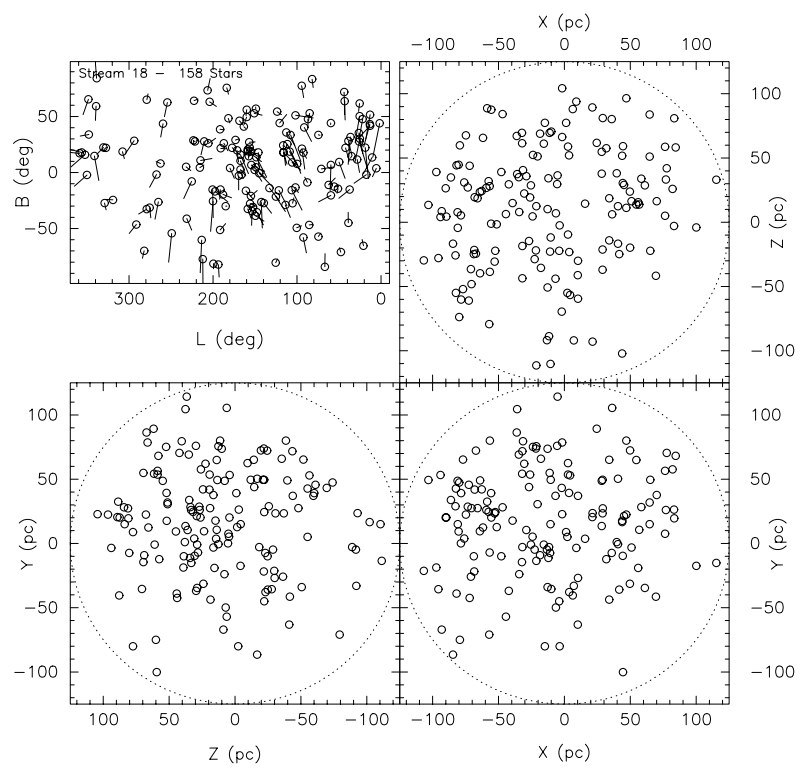

Fig. 26. Space distributions of New SCl (stream 3-18 in Table 2) from the $V_{R}$ selected sub-sample at scale 3

streams most of them corresponding to well defined age, a number of other streams are detected only at small scales. Stream $2-13$ in Table 3 (Fig. 27) is a typical example of such a stream. Its age distribution shows a mono-age component of $10^{9}$ year old and its vertical velocity is high $\left(W=-15.3 \mathrm{~km} \mathrm{~s}^{-1}\right)$. Space distribution does not fill the $125 \mathrm{pc}$ radius sphere and Fig. 27 probably shows on $(X, Z)$ and $(Y, Z)$ projections the orbit tube in which stars are confined. Stream 2-7 in Table 3 shows also the same features with a $610^{8}$ year old component and a lower vertical velocity $(W=$ $\left.-8.5 \mathrm{~km} \mathrm{~s}^{-1}\right)$.

- Particulars on oldest groups.

Another striking feature of the velocity field is the existence of a $210^{9}$ year old population still in velocity structures. These streams are only detected at the coarser resolution (scale 3) in agreement with an intrinsically large velocity dispersion. They have much less members (between 20 and 30 initial members) than the previously investigated streams and have very few observed $V_{R}$. Two main old groups (stream 3-9 and 3-14 in Table 2 and Figs. 28, 29) are clearly detected and have similar characteristics:

- Age distributions peak between 1.6 and $210^{9}$ yr.

- Their velocity dispersion obtained from the few selected stars on observed $V_{R}$ are of order $6 \mathrm{~km} \mathrm{~s}-1$ (stream 3-14 seems to have a lower velocity dispersion but the result is obtained for only 3 stars).

- U-component is positive (towards the galactic center) and often larger than $20 \mathrm{~km} \mathrm{~s}^{-1}$.

- Space distributions may still be clumpy. 

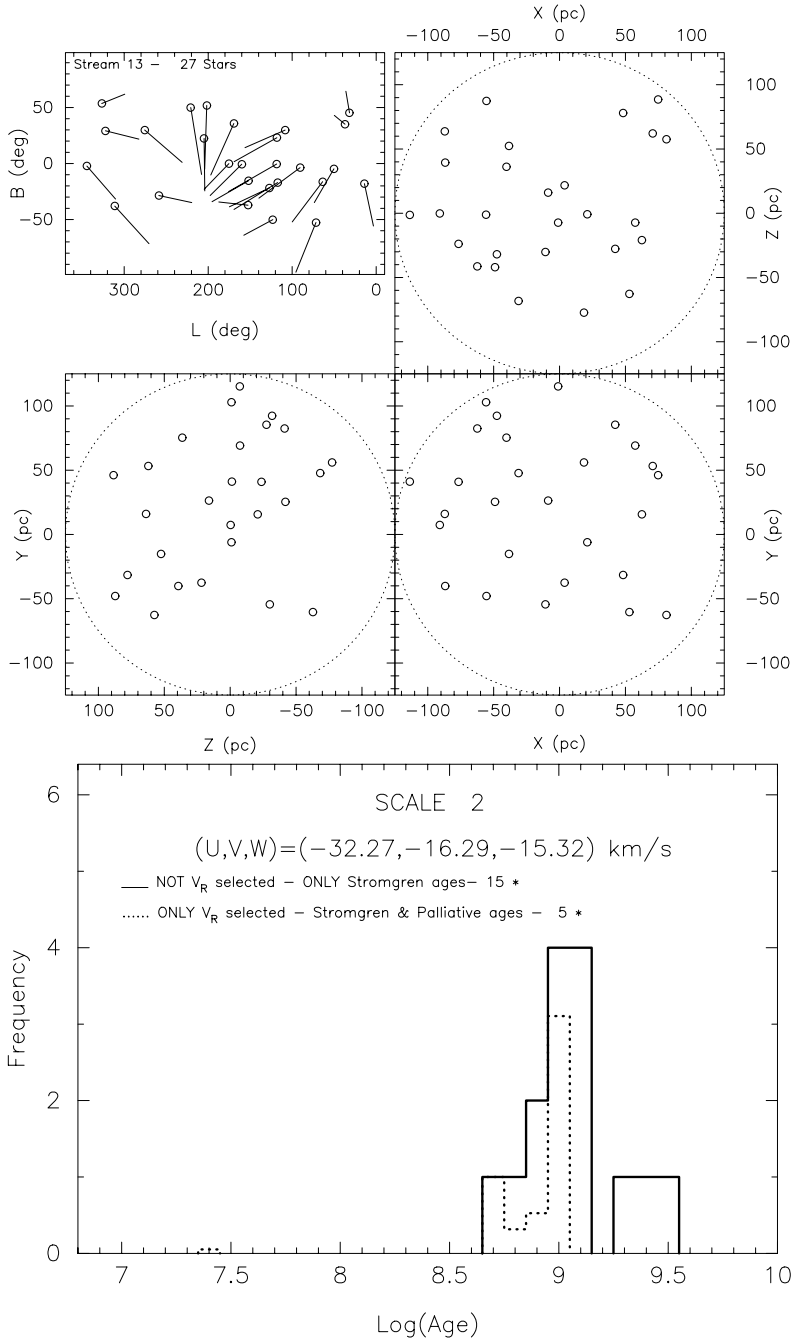

Fig. 27. Stream 2-13 (in Table 3). Space (top) and age (bottom) distributions from the sub-set without $V_{R}$ selection at scale 2

We should notice at this point that these old star streams are both affected by two biases with respect to the whole sample characteristics. They have much less observed radial velocities (16\% vs. $46 \%)$ and have much more Strömgren photometry ( $57 \%$ vs. $37 \%)$. For this reason, space distributions are displayed without radial velocity selection.

Streams are rather fragile structures originating from cluster evaporation. The survival of these relatively old moving groups with coherent ages can be explain by two non exclusive mechanisms. On one hand, recent simulations (Zwart et al. 1998) of heavy cluster dynamical evolution (with $\sim 32000$ particles) have shown that contrary to lighter open clusters whose typical lifetime is $1-210^{8}$ (Wielen 1971 and Lyngå 1982), heavy clusters can survive up to $4 \mathrm{Gyr}$ in the Galactic gravitational field. This long lifetime can authorize to maintain old streams but such heavy open clusters are probably very rare in the disc. On the other hand, as sketched in Dehnen (1998) to explain their eccentric orbits, stars of these moving
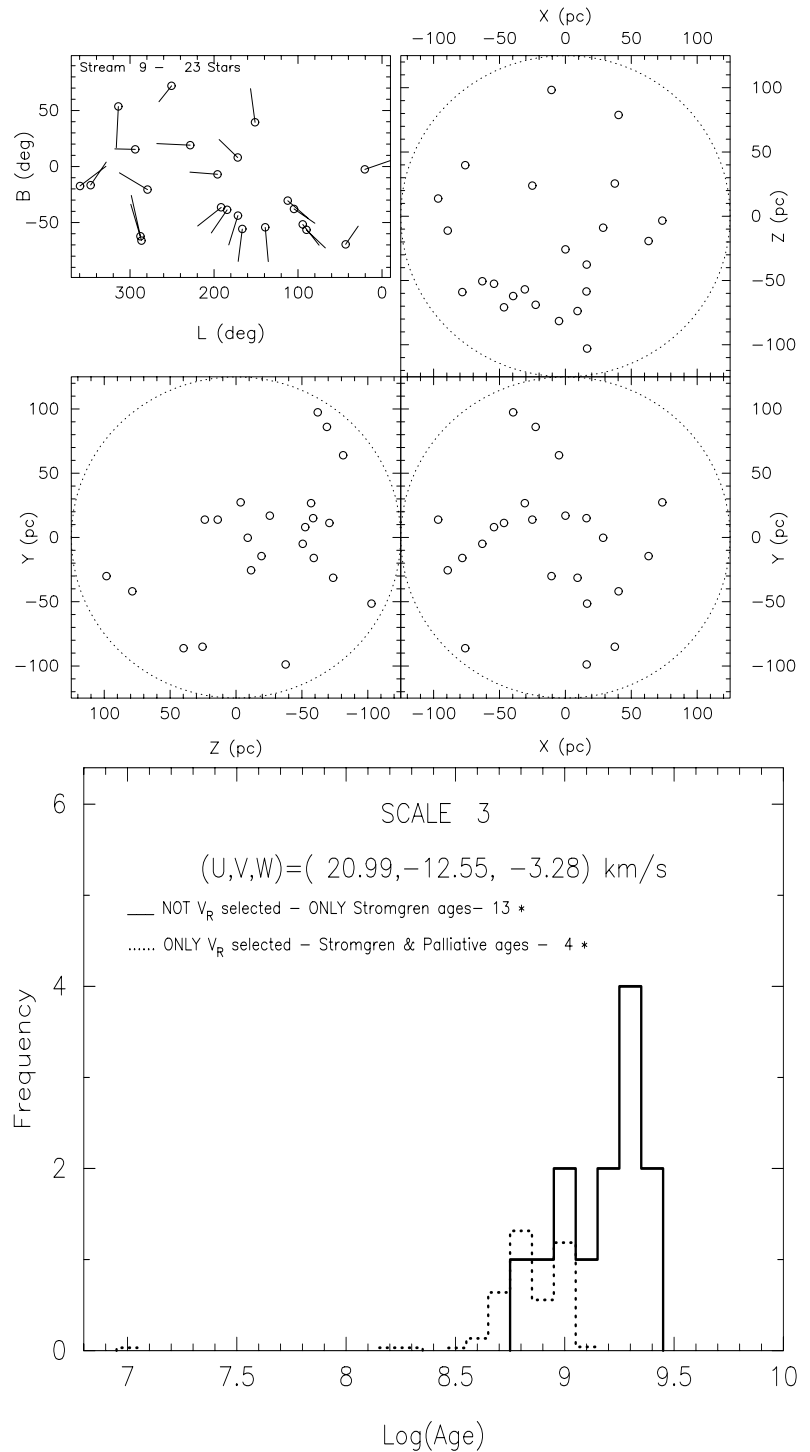

Fig. 28. Stream 3-9 (in Table 2). Space (top) and age (bottom) distributions from the sub-set without $V_{R}$ selection at scale 3

groups, provided that they were formed in the inner part of the disc, could have been trapped into resonant orbits with the non-axisymmetric force created by the gravitational potential of the Galactic bar. This latest explanation does not require any minimum lifetime to the initially bound structure from which streams originate.

\section{Conclusions}

A systematic multi-scale analysis of both the space and velocity distributions of a thin disc young star sample has been performed. Over-densities in space distributions are mainly well known open clusters (Hyades $\mathrm{OCl}$, Coma $\mathrm{OCl}$ and Ursa Major $\mathrm{OCl}$ ) and associations of stars (Centaurus-Crux and Centaurus-Lupus). Evidence 

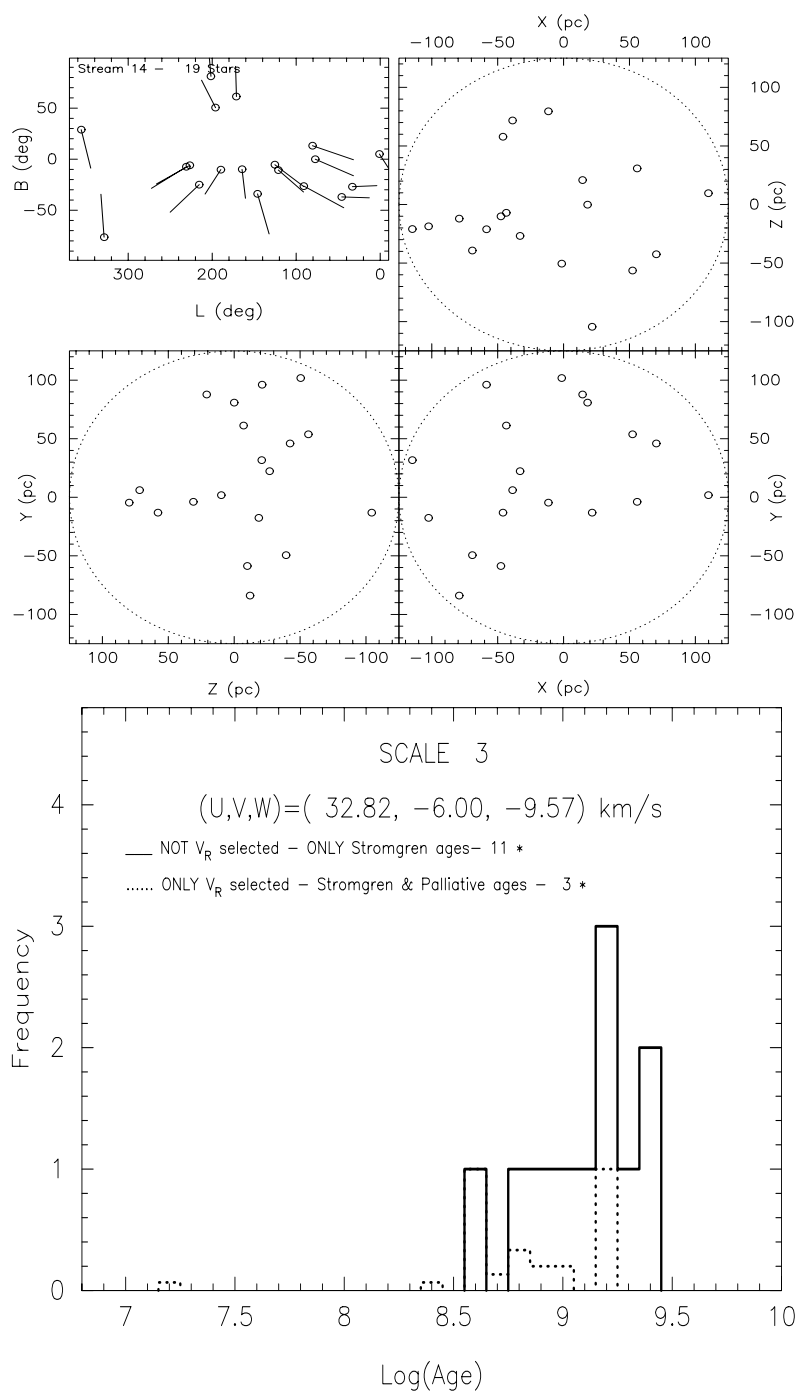

Fig. 29. Stream 3-14 (in Table 2). Space (top) and age (bottom) distributions from the sub-set without $V_{R}$ selection at scale 3

is given for three nearby loose clusters or associations not detected yet (Bootes and Pegasus 1 and 2). Evaporation of relatively massive stars $\left(1.8 M_{\odot}\right)$ out of the Hyades open cluster is also mapped in Paper II and form an asymmetric pattern. The sample is well mixed in position space since no more than $7 \%$ of the stars are in concentrated clumps with coherent tangential velocities.

The 3D velocity field reconstructed from a statistical convergent point method exhibits a strong structuring at typical scales of $\bar{\sigma}_{\text {stream }} \sim 6.3,3.8$ and $2.4 \mathrm{~km} \mathrm{~s}^{-1}$. At large scale (scale 3 ) the majority of structures are Eggen's superclusters (Pleiades SCl, Hyades $\mathrm{SCl}$ and Sirius SCl) with the whole Centaurus association embedded in a larger kinematic structure. A new supercluster-like structure is found with a mean velocity between the Sun and Sirius $\mathrm{SCl}$ velocities. These large scale velocity structures are all characterized by a large age range which reflects the overall sample age distribution. Moreover, few old streams of $\sim 2$ Gyr are also extracted at this scale with high $U$ components towards the Galactic center. Taking into account the fraction of spurious members, evaluated with an observed radial velocity data set, into all these large velocity dispersion structures we show that they represent $63 \%$ of the sample. This percentage drops to $46 \%$ if we remove the velocity background created by a smooth velocity ellipsoid in each structure. Smaller scales $\left(\bar{\sigma}_{\text {stream }} \sim\right.$ 3.8 and $2.4 \mathrm{~km} \mathrm{~s}^{-1}$ ) reveal that superclusters are always substructured by 2 or more streams which generally exhibits a coherent age distribution. The older the stream is the more difficult the age segregation between close velocity clumps is. At scale 2 and 1, background stars are negligible and percentages of stars in streams, after evaluating the fraction of spurious members, are $38 \%$ and $18 \%$ respectively.

Acknowledgements. This work was facilitated by the use of the Vizier service and the Simbad database developed at CDS. E.C thanks A. Bijaoui and J.L. Starck for constructive discussions on wavelet analysis, and C. Pichon for interesting discussions and help on some technical points. E.C is very grateful to R. Asiain for providing the two codes of age calculation from Strömgren photometry and thanks J. Torra and F. Figueras for valuable discussions.

\section{References}

Asiain R., Torra J., Figueras F., 1997, A\&A 322, 147

Bertelli G., Bressan A., Chiosi C., et al., 1994, A\&AS 106, 275

Chen B., Asiain R., Figueras F., Torra J., 1997, A\&A 318, 29

Chereul E., Crézé M., Bienaymé O., 1998, A\&A 340, 384 (Paper II)

Crézé M., Chereul E., Bienaymé O., Pichon C., 1998, A\&A 329, 920 (Paper I)

Dehnen W., 1998, AJ 115, 2384

Eggen O.J., 1973, PASP 85, 381

Eggen O.J., 1991, AJ 102, 2028

Eggen O.J., 1992a, AJ 103, 1302

Eggen O.J., 1992b, AJ 104, 1482

Eggen O.J., 1992c, AJ 104, 1493

Eggen O.J., 1992d, AJ 104, 2141

Eggen O.J., 1996, AJ 111, 1615

ESA, 1992, The Hipparcos Input Catalogue, ESA SP-1136

ESA, 1997, The Hipparcos Catalogue, ESA SP-1200

Figueras F., Torra J., Jordi C., 1991, A\&AS 87, 319

Figueras F., Gomez A.E., Asiain R., et al., 1997, Proceedings of the ESA Symposium "Hipparcos - Venice'97", ESA SP-402, p. 519

Holschneider M., Kronland-Martinet R., Morlet J., Tchamitchian P., 1989, in: Wavelets Combes J.M., et al. (eds.). Springer-Verlag, Berlin, p. 286

Lega E., Bijaoui A., Alimi J.M., Scholl H., 1996, A\&A 309, $23 \mathrm{~L}$

Lyngå G., 1982, A\&A 109, 213

Masana E., 1994, Tesis de Licenciatura

Pallous̀ J., Ruprecht J., Dluzhnevskaya O.B., Piskunov T., 1977, A\&A 61, 27

Pöppel W., 1997, Fundamental of Cosmic Physics, Vol. 18

Turon C., et al., 1992, A\&A 258, 74

Wielen R., 1971, A\&A 13, 309

Zwart S.F.P, Hut P., Makino J., McMillan S.L.W., 1998, (submitted to A\&A) 